INRA Prod. Anim., 2017, 30 (4), 303-320

\title{
Diversité des services rendus par les territoires à forte densité d'élevages, trois cas d'étude en Europe
}

\author{
J.-Y. DOURMAD ${ }^{1}$, L. DELABY $Y^{1}$, J.-L. BOIXADERA', \\ C. ORTIS ${ }^{2}$, B. MÉDA $A^{3}, C . G A I G N E^{4}, B . D U M O N T^{5}$ \\ ${ }^{\prime}$ PEGASE, Agrocampus Ouest, INRA, 35590, Saint-Gilles, France \\ 2 Gouvernement de Catalogne, 25198, Lleida, Espagne \\ ${ }_{3}$ URA, INRA, 37380, Nouzilly, France \\ 4 UMR 1302 SMART-LERECO, Agrocampus Ouest, INRA, 35000, Rennes, France \\ ${ }_{5}$ Université Clermont Auvergne, INRA, Vetagro Sup, UMR Herbivores, 63122, Saint-Genès-Champanelle, France \\ Courriel : Jean-Yves.Dourmad@inra.fr
}

Les territoires à forte densité d'élevages se caractérisent par une production élevée par unité de surface et par unité de travail, avec un recours important à des intrants, surtout pour l'alimentation des animaux. Dans cet article, nous illustrons les bouquets de services rendus par l'élevage dans trois territoires européens différenciés par le contexte de production, la sensibilité des milieux et les choix stratégiques et technologiques mis en œuvre pour la gestion des effluents.

Les territoires en tension du fait d'une forte concentration animale sont caractérisés par une production élevée de produits animaux par hectare et une charge élevée en effluents d'élevage, au regard des surfaces disponibles pour l'épandage. Au niveau européen, la directive Nitrate s'applique dans toutes les zones dites « vulnérables », où les eaux souterraines ou superficielles présentent des teneurs élevées en nitrates. Cette directive limite les épandages d'azote d'origine animale à $170 \mathrm{~kg}$ par ha et par an. Au milieu des années 90, différentes pratiques ont été mises en place pour respecter ce plafond avec des stratégies variables selon les pays comme :

i) le traitement des excès d'azote (par exemple en France);

ii) l'exportation d'azote vers des exploitations ou des régions déficitaires, parfois dans le cadre de plan d'épandage collectifs ou de « banques à lisier » (aux Pays-Bas, en Allemagne et en Catalogne) ;

iii) le développement de stratégies innovantes de valorisation des effluents comme la méthanisation (en Allemagne et au Danemark) ou la production d'engrais organiques (aux Pays-Bas ou en France).
Ces différents choix stratégiques sont en partie la conséquence des modalités politico-administratives de mise en œuvre de la directive Nitrate dans chaque pays. Une réglementation sur les émissions d'ammoniac et l'épandage de phosphore d'origine animale a également progressivement été mise en place dans la plupart des pays.

Pour illustrer ces situations, nous avons retenu trois territoires à forte densité d'élevage qui se distinguent par le contexte (densité et diversité des élevages), la sensibilité des milieux et les choix stratégiques et technologiques mis en œuvre pour gérer les effluents :

i) la Bretagne, un territoire de polyculturepolyélevage avec une forte densité de productions animales et des zones particulièrement sensibles sur le plan environnemental ;

ii) le nord de l'Allemagne, avec la particularité d'un très fort développement de la méthanisation à des fins de production d'énergie renouvelable ;

iii) la Catalogne, un territoire marqué par un développement récent et rapide de la production porcine dans un milieu où les surfaces agricoles disponibles pour l'épandage sont limitées.

\section{1 / La Bretagne, un territoire de polyculture-polyélevage avec une forte densité de production}

\section{1 / Contexte}

a) L'élevage un secteur clé de l'agriculture bretonne

En Bretagne, l'agriculture et l'agroalimentaire sont des secteurs très importants sur le plan économique et en termes d'emplois. L'élevage en constitue le secteur dominant : sept exploitations bretonnes sur 10 ont une activité spécialisée dans l'élevage (Agreste Bretagne, 2014). La SAU représente $62 \%$ de la surface totale du territoire, soit une valeur un peu plus élevée que la moyenne nationale (54\%) (tableau 1). Les surfaces toujours en herbe représentent $11 \%$ de la SAU. Cette valeur est inférieure à la moyenne nationale mais elle sous-estime la surface réellement en herbe du fait de la présence significative de prairies cultivées et pâturées dans les rotations culturales. Au total, la surface en herbe représente $43 \%$ de la SAU, une valeur identique à la moyenne nationale. Les surfaces en céréales et oléoprotéagineux couvrent $35 \%$ de la SAU auxquels viennent s'ajouter environ $19 \%$ de maïs ensilage. La production de maïs 
Tableau 1. Utilisation des surfaces en Bretagne et dans de Grand Ouest de la France, en comparaison à la moyenne française (D'après Agreste Bretagne 2014).

\begin{tabular}{|l|c|c|c|c|c|c|c|c|}
\hline & \multicolumn{2}{|c|}{$\%$ de la surface } & \multicolumn{9}{|c|}{ Répartition de la SAU (\%) } \\
\cline { 2 - 9 } & SAU & $\begin{array}{c}\text { Forêt et } \\
\text { landes }\end{array}$ & $\begin{array}{c}\text { Céréales } \\
\text { et oléo- } \\
\text { protéagineux }\end{array}$ & $\begin{array}{c}\text { Maïs } \\
\text { ensilage }\end{array}$ & $\begin{array}{c}\text { Prairie } \\
\text { permanente }\end{array}$ & $\begin{array}{c}\text { Surface } \\
\text { totale en } \\
\text { herbe }\end{array}$ & $\begin{array}{c}\text { Surface en } \\
\text { production } \\
\text { biologique }\end{array}$ & $\begin{array}{c}\text { Viticulture, } \\
\text { fruits, } \\
\text { légumes }\end{array}$ \\
\hline Bretagne & 62 & 24 & 35,1 & 19,2 & 11,5 & 42,8 & 2,9 & 3,0 \\
Grand Ouest $^{(1)}$ & 68 & 16 & 32,8 & 14,8 & 23,8 & 47,0 & 3,6 & 2,1 \\
\hline France & 53 & 36 & 47,5 & 4,9 & 31,4 & 43,4 & 2,9 & 3,9 \\
\hline
\end{tabular}

${ }^{(1)}$ Le Grand Ouest regroupe les régions Bretagne, Pays de Loire et Basse-Normandie.

Tableau 2. Contribution de la Bretagne et du Grand Ouest à la production nationale de produits animaux, lait, viande et œufs. D’après Agreste Bretagne (2014).

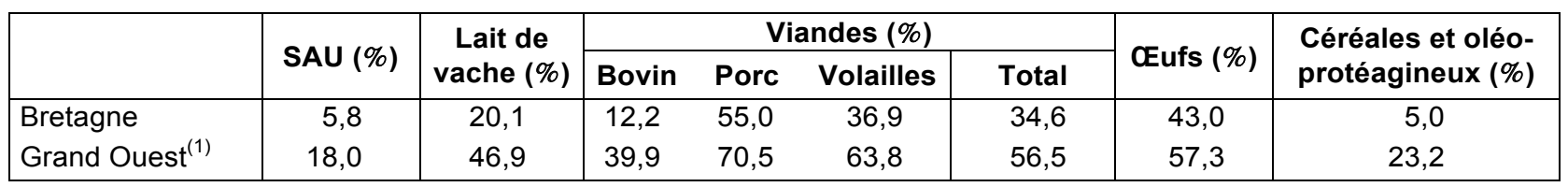

${ }^{(1)}$ Le Grand Ouest regroupe les régions Bretagne, Pays de Loire et Basse-Normandie.

ensilage est importante en Bretagne et représente $23 \%$ de la production nationale. La Bretagne est aussi la première région légumière française. Enfin, la particularité de la Bretagne est la présence simultanée de trois filières (porcs, lait et volaille) tandis que les autres régions européennes à forte densité animale sont généralement plus spécialisées dans une ou deux filières animales (Gaigné et Letort 2017) même si des situations similaires se retrouvent également dans d'autres régions Européennes (Roguet et al 2015).

\section{b) Les facteurs historiques de l'évolution de l'élevage}

Les facteurs historiques qui ont conduit à cette situation ont évolué au cours du temps. Les grandes étapes sont illustrées à la figure 1 pour les filières laitière, porcine et avicole (Draaf Bretagne 2013). La faible taille des exploitations, la forte population rurale au sortir de la seconde guerre mondiale et la volonté de « travailler au pays » ont favorisé dès les années 1960 le développement des élevages « hors-sol » (volailles et porcs) parallèlement à celui de l'élevage laitier (Draaf Bretagne 2013). La mise en place des quotas laitiers en 1984, sans la possibilité de les transférer géographiquement, a stoppé la dynamique de développement de la production laitière et a contribué à renforcer le développement des céréales, des productions de monogastriques, surtout le porc, et dans une moindre mesure celui des troupeaux allaitants. Les évolutions ultérieures ont été favorisées par des économies d'échelles, l'augmentation de la taille des élevages et leur spécialisation permettant d'améliorer leur efficacité, y compris pour trouver des solutions aux problèmes environnementaux (Gaigné et al 2012), et leur concentration géographique permettant d'améliorer l'efficacité technique et économique des filières. L'apogée du développement de la filière avicole a été atteint en 1990, la production ayant régressé par la suite. Pour la filière porcine, l'apogée a été atteinte en 2000, la production s'étant ensuite stabilisée pour commencer à diminuer ces dernières années.

\section{c) Des filières animales présentes sur tout le territoire}

Sur le plan géographique, les différentes productions animales sont présentes sur l'ensemble du territoire avec toutefois quelques nuances et spécialisations locales. La production porcine est plus présente dans les Côtes d'Armor et le Finistère, la production laitière en Illeet-Vilaine et la production avicole dans le Morbihan. Par contre, les exploitations se sont de plus en plus spécialisées. Selon le dernier recensement agricole de 2010, $70 \%$ d'entre elles sont classées dans les orientations technico-économiques (Otex) « Bovin lait» $(38 \%)$ ou « Élevage hors sol » $(31 \%)$.

\section{d) Un élevage dépendant de l'extérieur pour l'alimentation des animaux}

Cette forte densité animale est associée à une forte utilisation d'intrants, en particulier pour l'alimentation des animaux. En 2013, le déficit en matières premières pour l'alimentation animale est estimé à 5,6 millions de tonnes soit un peu plus que la totalité de la production régionale qui avoisine de 5 millions de tonnes (Nutrinoe 2015). Les céréales représentent la part principale de ce déficit (40\%), suivi du tourteau de soja (18\%), du tourteau de colza (14\%), des coproduits des céréales $(10 \%)$ et du tourteau de tournesol $(7 \%)$. En 2013, la Bretagne a ainsi importé environ un million de tonnes de tourteau de soja dont environ $45 \%$ pour les volailles, $35 \%$ pour les ruminants et $20 \%$ pour les porcs (Nutrinoe 2015). Cette valeur est en baisse de 600000 tonnes par rapport à 2003, au profit de l'utilisation du tourteau de colza et de tournesol, et d'acides aminés de synthèse. Sur la base du critère " utilisation de surface » disponible pour les différentes matières premières utilisées en alimentation animale en France (Wilfart et al 2016), on peut estimer que les matières premières importées correspondent à une SAU d'environ 670000 ha, soit l'équivalent de $40 \%$ de la SAU de la Bretagne, dont environ 250000 ha correspondraient à des cultures situées en dehors de l'UE (principalement du soja au Brésil et du tournesol dans la région de la Mer Noire).

\section{2 / Services d'approvisionnement}

a) Une contribution majeure à la production de protéines animales au niveau national

En termes de services d'approvisionnement, la principale caractéristique de l'élevage de cette région est sa très forte contribution à l'approvisionnement national en produits animaux et en protéines pour l'alimentation humaine. Avec une surface agricole qui représente seulement $5,8 \%$ de la SAU nationale, la Bretagne réalise en effet $20 \%$ de la production de lait du pays, $43 \%$ des œufs, $12 \%$ de la viande bovine, $55 \%$ de la viande porcine et $37 \%$ de la viande de volaille, soit $35 \%$ de la production nationale de viandes (tableau 2) ou un tiers de la consommation nationale de protéines animales (poisson non compris). 
Figure 1. Les grandes étapes du développement des filières de production de lait, porc et volailles en Bretagne (Draaf Bretagne, 2013).

\section{Filière laitière}

1984 Mise en place des quotas laitiers

2003 Réformes PAC et OCM lait 2015 Fins des quotas

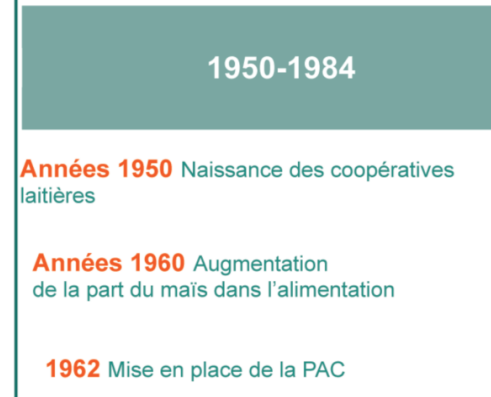

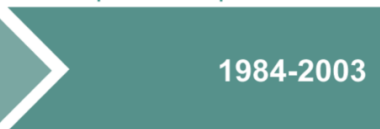

1990 Prise de conscience de l'impact de l'agriculture sur l'environnement

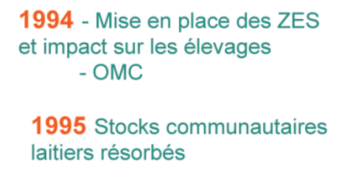

1995 Stocks communautaires laitiers résorbés

1997 Premiers accords

interprofessionnels sur les

recommandations du prix du lait

De la régulation à la sortie des quotas

\section{3-2015}

2004 Aide directe versée aux producteurs

2006 Découplage des aides du 1er pilier de la PAC

2012 « Paquet lait » de la PAC

2013 Huit premières

organisations de producteurs

laiters reconnues par la MAAF

Reconstruction de l'Europe

Libéralisation de la filière

\section{Filière Porcine}

1983-1984 Première crise de la filière

2000 Apogée de la production porcine bretonne

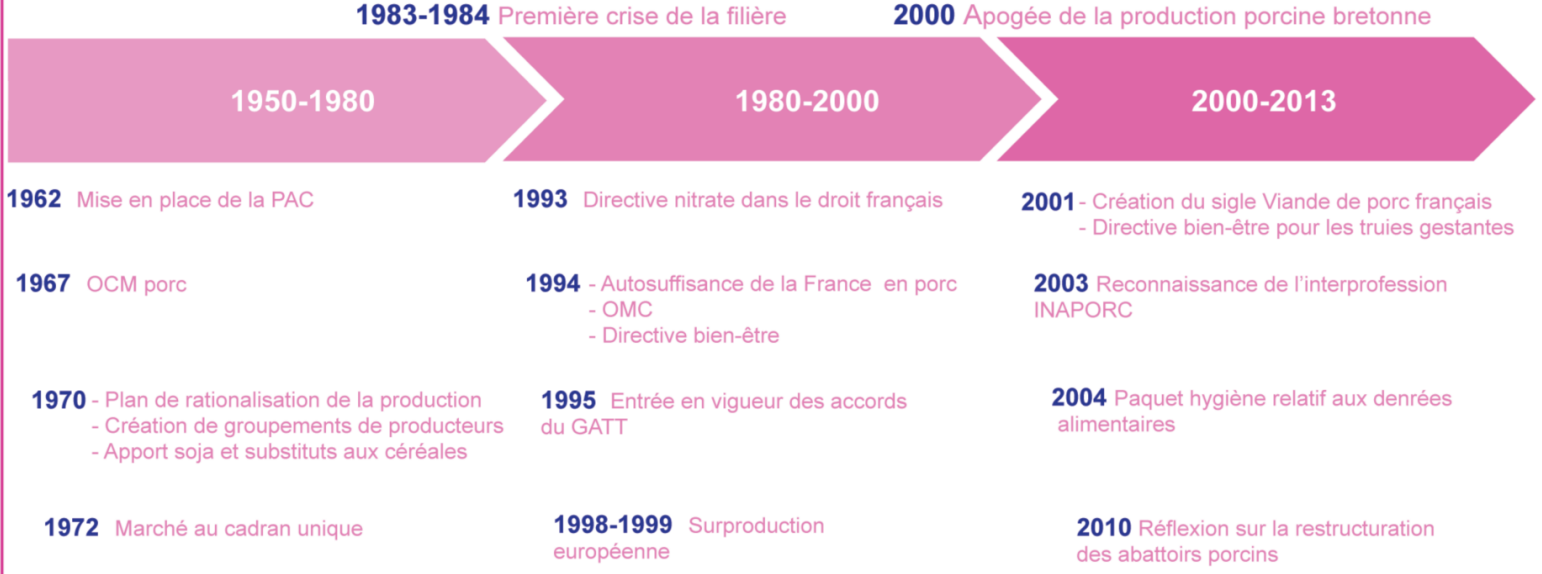

1974 Uniporc

Une des filières délevage les moins soutenues par la PAC... et pourtant

\section{Rationalisation et productivité}

\section{Sous la contrainte}

\section{Filière Volailles de chair}

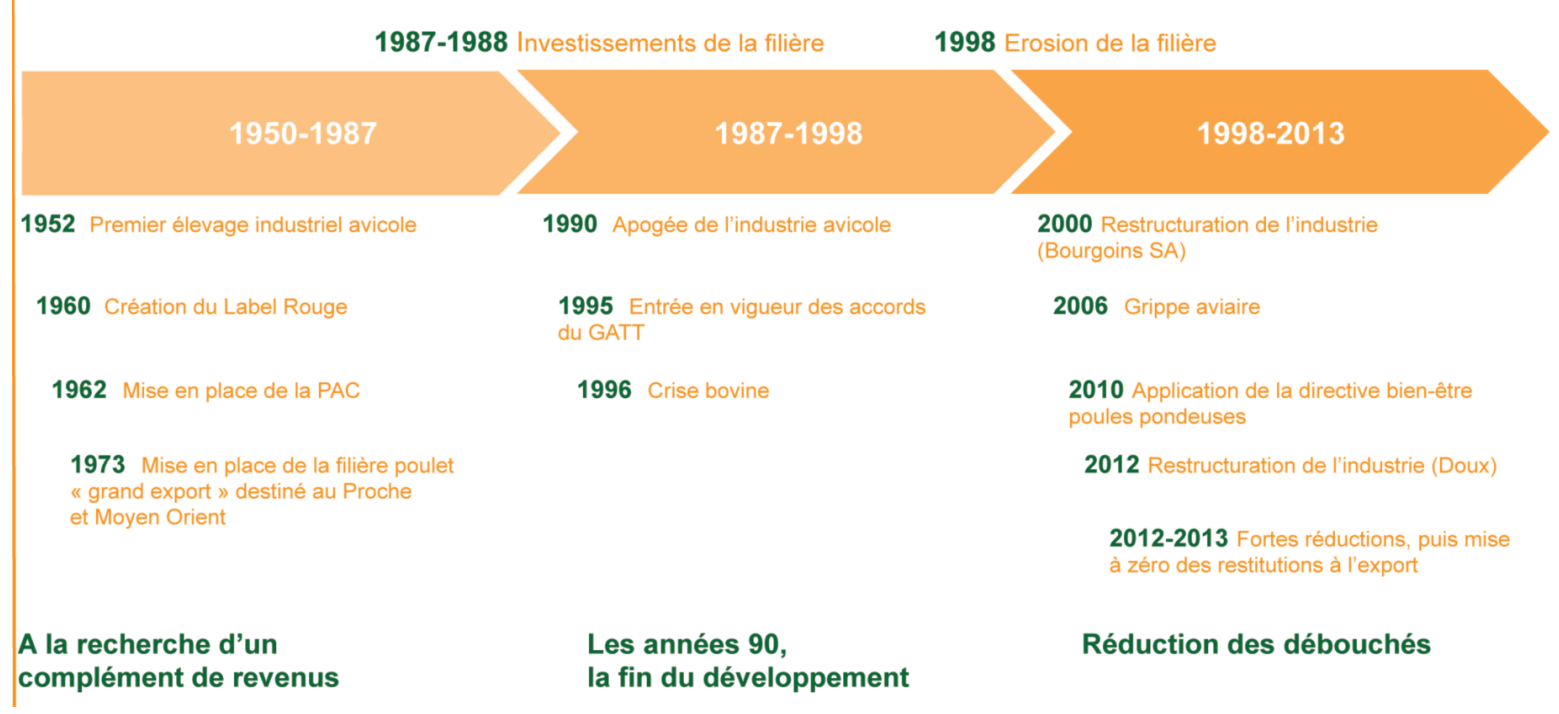


b) Des systèmes d'élevages et des produits moins diversifiés que sur le reste du territoire national

Une part significative des élevages de porcs bretons s'inscrit dans des filières de qualité et bénéficie d'une labellisation 2013). Le label le plus représenté, mais aussi le moins contraignant, est le label « le Porc Français » puisque près de $90 \%$ des élevages sont certifiés. Le label CCP (Certificat Conformité Produit) constitue le deuxième label le plus représenté avec $46 \%$ des élevages. La certification Label Rouge concerne 3\% des élevages de la région mais, avec environ $22 \%$ de la production nationale, la Bretagne est la première région productrice. Enfin, la Bretagne représente environ $16 \%$ de la production nationale de porc bio, derrière les Pays de la Loire. Ceci ne concerne toutefois qu'une faible proportion de la production puisqu'au niveau national, le porc bio ne représente qu'environ $0,4 \%$ de la production française en 2015 et de l'ordre de $0,15 \%$ en Bretagne.

En 2013, la Bretagne a produit $36 \%$ de la production française de poudre de lait, $26 \%$ de la production de beurre, $19 \%$ de la production de crème, $18 \%$ de la production de lait liquide, $13 \%$ de la production de fromage (pour moitié de fromage à pâte pressée cuite de type emmental) et $4 \%$ de la production de yaourt (Béline et al 2010). La fabrication de ces différents produits laitiers est en progression sauf pour la poudre de lait et les yaourts. Positionnée au deuxième rang derrière les Pays de la Loire, la Bretagne fournit $22 \%$ de la collecte nationale de lait bio en 2014 ; $60 \%$ du beurre bio et $42 \%$ des crèmes conditionnées bio sont fabriqués en Bretagne.

La production de volailles est relativement diversifiée en termes d'espèces. La Bretagne produit respectivement 32, 42,17 et $8 \%$ de la production nationale de poulet, de dinde, de canard à rôtir et de pintade. Elle produit $43 \%$ de la production nationale d'œufs et $23 \%$ de la production de lapin. La production de dinde et surtout de poulet pour l'exportation, en particulier vers les pays du Golfe, reste significative malgré d'importantes restructurations liées à l'abandon des restitutions. En 2014, les exportations bretonnes de volailles vers les pays tiers s'élevaient ainsi à 275 millions d'euros, compensant ainsi une part très significative des importations, principalement en provenance de l'UE, pour la consommation nationale, avec au final une balance commerciale positive au niveau national (Chatellier et al 2015).

\section{3 / Services pour l'environnement}

a) Des problèmes environnementaux persistent malgré une situation générale qui s'améliore

Le développement des productions animales a été à l'origine, dès la fin des années 1980, de l'apparition de problèmes environnementaux, résultant de la trop forte concentration des élevages et de la proximité d'un littoral particulièrement sensible (eaux peu profondes dans des baies fermées ou confinées limitant le brassage de l'azote dans l'eau). Cela a conduit à une remise en cause par la société des modèles intensifs d'élevage en raison des problèmes environnementaux et sanitaires dont certains sont directement " perceptibles » par les citoyens (teneur en nitrate de l'eau du robinet, algues vertes, odeurs). Des règlementations visant à réduire l'impact environnemental des élevages sont alors apparues, la directive nitrate étant la plus connue (Le Goffe 2008). La totalité de la région a été placé en zone sensible et environ 52\% des cantons (en 2001) en Zone d'Excédents Structurels (ZES) (Gaigné 2012).

Cette situation a conduit à la mise en œuvre de différentes pratiques permettant de réduire dans chaque exploitation la charge azotée d'origine animale en dessous de $170 \mathrm{~kg} \mathrm{~N} / \mathrm{ha}$. Les principales mesures ont dans un premier temps consisté à $i$ ) réduire à la source l'entrée d'azote par l'alimentation, ii) traiter les déjections pour réduire leur teneur en $\mathrm{N}$ (digestion aérobie, compostage ; 445 stations de traitement en 2014), iii) transférer les effluents vers des cantons hors ZES, iv) améliorer les pratiques d'épandage pour accroître la surface épandable (enfouissement, atténuation des odeurs) (Gaigné 2012). La réduction à la source par l'alimentation et le traitement des déjections ont constitué la principale voie de résorption pour la filière porcine, la filière avicole privilégiant l'exportation hors zone, les fientes et fumiers étant plus faciles à sécher et à transporter. Les différentes filières de traitement ont également conduit à la production de fertilisants organiques (115000 tonnes en 2011) commercialisés auprès des arboriculteurs, maraîchers, viticulteurs, etc. La réduction du cheptel de volailles, dans une moindre mesure de bovins, et plus récemment de porcs a également contribué à réduire la quantité d'azote organique. Parallèlement, l'utilisation d'azote minéral a également été réduite avec la mise en place des plans de fertilisation prévisionnels et par la montée des prix des engrais minéraux en 2008 et 2012-2013 (Gaigné 2012).

Ces différentes évolutions ont conduit à une amélioration de la balance azotée globale dont l'excédent est passé de près de $60 \mathrm{~kg}$ par ha de SAU en 2001 à $29 \mathrm{~kg}$ en 2014. Ceci s'est répercuté sur les teneurs en nitrate des eaux de surface. Après avoir fortement augmenté sur la période 1980-2000, ces teneurs baissent depuis 2000. En 2013, seuls quelques points de prélèvement avaient une teneur supérieure à la référence pour la consommation humaine $(50 \mathrm{mg} / \mathrm{L}$, figure 2$)$. $\mathrm{La}$ situation s'est également beaucoup améliorée pour la qualité des eaux douces en termes de risque d'eutrophisation, qui est principalement liée à la teneur en phosphore. En 2013, plus de $95 \%$ des sites de prélèvement sont classés " bon » ou « très bon ». La situation est moins satisfaisante pour la teneur en matière organique dissoute puisque seulement $45 \%$ des sites se classent en bonne qualité ou très bonne qualité, l'est de la région présentant une situation beaucoup moins favorable que l'ouest (Dreal 2014).

b) Des problèmes qui persistent dans des zones plus sensibles

Malgré ces progrès, le constat peut cependant toujours être fait aujourd'hui de la persistance d'une image dégradée dans la population et les médias des productions animales, surtout porcines et avicoles. Cette situation est en grande partie liées à la persistance de zones localisées d'échouages d'algues vertes qui s'expliquent par l'existence de masses d'eau côtière de faible profondeur et peu turbide sur une large étendue, un confinement hydrodynamique de cette masse d'eau côtière et l'arrivée d'un flux significatif d'azote apporté par les cours d'eau (Menesguen 2003). La majorité de ces échouages ont lieu en période estivale avec toutefois une forte variabilité inter annuelle. C'est la situation par exemple de la baie de Saint-Michel-enGrève (Côtes d'Armor) qui est affectée, depuis les années 1970, par des «marées vertes 》 récurrentes malgré des concentrations moyennes en nitrate des cours d'eau modérées (inférieure à $30 \mathrm{mg} / \mathrm{L}$ ), dans un contexte agricole dominé par des exploitations de bovins laitiers qui représentent $88 \%$ des exploitations du bassin versant (Mabon et al 2009). L'étude des systèmes existants dans le cadre d'un programme ANR (http:// www6.inra.fr/acassya) montre qu'il existe des marges de progrès dans la gestion de l'azote et que certaines exploitations ont réussi à mettre en application des stratégies économes qui s'accompagnent de bons résultats économiques et environnementaux (Mabon et al 2009). Les simulations indiquent cependant que l'adoption de mesures agroenvironnementales « MAE Système fourrager économe en intrants » sur l'ensemble du bassin versant ne suffirait pas pour atteindre les objectifs de qualité de l'eau (Moreau et al 2012). Après dix ans d'ap- 
Figure 2. Évolution de la concentration en nitrate des eaux de surface (percentile 90) entre 1980 et 2013 et répartition géographique des résultats pour l'année 2013 (Dréal Bretagne, 2014).
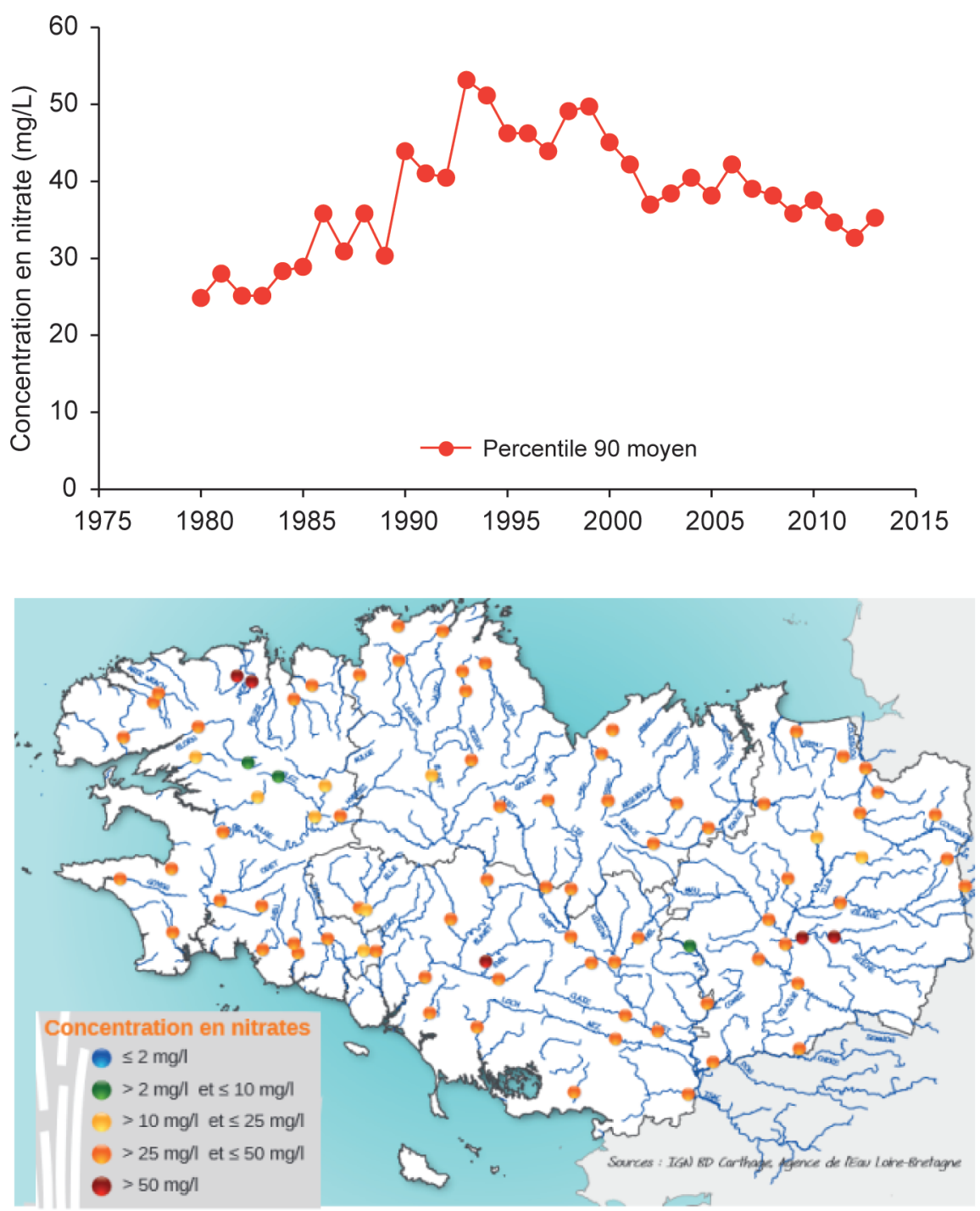

plication la réduction de teneur en nitrate entraînée par les changements de pratiques ne serait que de l'ordre de $3 \mathrm{mg} / \mathrm{L}$, comparativement à la poursuite des pratiques actuelles, soit une réduction du lessivage de seulement $2 \mathrm{~kg} \mathrm{~N} / \mathrm{ha} / \mathrm{an}$ pour une diminution des apports de $24 \mathrm{~kg} \mathrm{~N} / \mathrm{ha} / \mathrm{an}$.

c) Des systèmes d'élevage efficaces en termes d'émissions de Gaz à Effet de Serre (GES) par kg de protéines animales

La production de GES de l'élevage en Bretagne s'élève à environ $40 \mathrm{~kg}$ éq $\mathrm{CO}_{2}$ par kg de protéines animales produites pour l'alimentation humaine (calculé d'après Weiss et Leip 2012). Cette valeur est nettement plus faible que la moyenne européenne estimée à $62 \mathrm{~kg}$ et encore plus faible que celles des zones plus extensives d'élevage (plus de $80 \mathrm{~kg}$ éq $\mathrm{CO}_{2}$ par $\mathrm{kg}$ de protéines). Ceci s'explique par des systèmes d'élevage principalement orientés vers la production de lait, d'œufs et de viande de volaille et de porc, sages à l'échelle des régions, la Bretagne se situe dans le quart supérieur des régions les plus diversifiées avec un indice de Shannon ( « Shannon Evenness Index ») supérieur à 0,72 (contrairement par exemple à l'Irlande, qui se situe parmi les régions les moins diversifiées). L'élevage a également contribué à la préservation d'un paysage de bocage et de haies même si la tendance générale est à la baisse, en particulier dans l'est de la région. Une étude conduite en 2010 (Agreste Bretagne 2010) évalue ainsi à $185000 \mathrm{~km}$ la longueur du linéaire bocager de Bretagne, soit en moyenne $110 \mathrm{~m} / \mathrm{ha}$ SAU. Comme dans les autres régions de bocage en Europe, l'évolution de l'agriculture depuis les années 1950 s'est accompagnée d'un éclaircissement du bocage favorisé aussi par le remembrement (Thenail 2002). D'après Thenail (2002), la situation actuelle du bocage résulterait de la co-évolution des systèmes agricoles et des paysages, qui constitueraient alors une des forces motrices du développement agricole. La diversité des « styles » d'agriculteurs et d'éleveurs contribuerait alors à expliquer la coexistence, à proximité, de zones de bocage dense ou plus ouvert. La densité apparente du bocage n'est toutefois pas à elle seule garante d'effets positifs pour l'environnement, ces derniers étant aussi largement dépendant des pratiques de gestion des parcelles et des bordures, sur le court et le long terme. Un bocage trop dense peut même parfois constituer un frein à la mise en œuvre de bonnes pratiques (Thenail 2002).

Par ailleurs, il convient de rappeler que les productions animales bretonnes restent en partie dépendantes, pour la couverture des besoins en protéines, des importations de soja en provenance d'Amérique du Sud. Ceci a des effets induits sur la biodiversité en particulier lorsque l'extension de ces cultures entraîne de la déforestation, même si cette dernière tend à diminuer fortement ces dernières années du fait du moratoire brésilien (Gibbs et al 2015).

\section{4 / Impacts socio-économiques}

La forte densité animale permet une production élevée par unité de surface, favorisant ainsi le développement des activités amont (alimentation animale, équipements d'élevage) et aval (transformations des produits) dont la densité est étroitement liée à celle de l'élevage. Elle s'accompagne d'un nombre d'actifs agricoles élevé par unité de surface (3,3 UTA/100 ha SAU). À titre de comparaison, les valeurs correspondantes dans des régions céréalières (Centre) ou d'élevage plus extensif (Auvergne) sont respectivement de 1,6 et 2,1 UTA/100 ha SAU. 
Figure 3. Représentation graphique du cas d'étude " polyculture-polyélevage de la région Bretagne » selon le formalisme de la "grange ".

Comme décrit par Duru et al (2017), le pentagone central représente un territoire d'élevage avec ses éléments paysagers (haies) et les activités agro-industrielles (IAA, méthaniseur) sur lesquelles s'appuie l'élevage. Celui-ci est décrit par ses caractéristiques : espèces et densité animales, modes d'alimentation (animal vert : nourri au pâturage et à base de fourrages, ocre : aliments concentrés dont l'ensilage de maïs). Le mode d'usage des terres est représenté par un parcellaire à deux nuances de vert pour symboliser la diversité des prairies permanentes et la présence de temporaires, et à deux nuances de jaune pour représenter la diversité des rotations culturales. Au sein du territoire, les systèmes d'élevage interagissent avec cinq interfaces. Les pictogrammes explicités dans le tableau joint symbolisent les éléments concernés par ces interfaces. La nature et l'ampleur des effets sont représentées par une flèche sortante plus ou moins large et dont la couleur indique que ceux-ci sont positifs (flèche verte), négatifs (rouge) ou mitigés (hachures) ; dans ce cas, l'effet dominant borde la flèche. Sur l'interface travail et emploi, nous distinguons les emplois directs en élevage des emplois indirects (IAA, tourisme, flèche vers personnage blanc). Les flèches entrantes indiquent l'utilisation d'intrants exogènes (qui symbolisent les effets délocalisés de l'élevage), des pressions ou des opportunités liées aux marchés, ou (sur l'interface environnement et climat) que l'élevage bénéficie de services intrants (flèche verte).

Tableau 3. Impacts positifs et négatifs associés aux territoires de polyculture-polyélevage de la région Bretagne.

\begin{tabular}{|c|c|c|}
\hline $\begin{array}{l}\text { Interfaces } \\
\text { étudiées }\end{array}$ & Effets positifs & Effets négatifs \\
\hline Intrants & $\begin{array}{l}\text { - Recyclage des effluents organiques comme } \\
\text { fertilisants } \\
\text { - Importation de coproduits (déchets de } \\
\text { biscuiteries...) }\end{array}$ & $\begin{array}{l}\text { - Effets délocalisés (usage des terres, énergie, } \\
\text { pesticides) pour la production des concentrés } \\
\text { - Utilisation d'engrais et de pesticides }\end{array}$ \\
\hline $\begin{array}{l}\text { Travail } \\
\text { et emploi }\end{array}$ & $\begin{array}{l}\text { - Nombreux emplois dans des filières } \\
\text { agroalimentaires bien organisées } \\
\text { et dans les activités d'amont (équipement, } \\
\text { bâtiments, alimentation animale) } \\
\text { - Nombreux emplois directs dans les élevages }\end{array}$ & $\begin{array}{l}\text { - Questionnements sur la compétitivité des filières } \\
\text { et les conditions de travail dans l'agroalimentaire }\end{array}$ \\
\hline Marchés & $\begin{array}{l}\text { - Production de masse à prix très compétitifs } \\
\text { - Contribution significative à la demande nationale } \\
\text { - Exportation vers les pays de l'UE et les pays tiers }\end{array}$ & $\begin{array}{l}\text { - Dépendance vis-à-vis de la conjoncture } \\
\text { internationale (prix des matières premières } \\
\text { et mise sur le marché) et coût du travail }\end{array}$ \\
\hline $\begin{array}{l}\text { Enjeux sociaux } \\
\text { et culturels }\end{array}$ & $\begin{array}{l}\text { - Acceptabilité locale liées aux emplois créés } \\
\text { - Contribution au maintien du dynamisme } \\
\text { des territoires ruraux }\end{array}$ & $\begin{array}{l}\text { - Risques accrus pour la santé animale du fait } \\
\text { de densités élevées } \\
\text { - Questionnement sur le bien-être des animaux, } \\
\text { les nuisances olfactives, la pollution de l'air } \\
\text { et de l'eau, l'antibiorésistance }\end{array}$ \\
\hline $\begin{array}{l}\text { Environnement } \\
\text { et climat }\end{array}$ & $\begin{array}{l}\text { - Faibles émissions directes et indirectes de GES } \\
\text { par kg de produit } \\
\text { - Services intrants ( } \mathrm{N} \text { fixé par les légumineuses, } \\
\text { bocage, maintien de paysages diversifiés) }\end{array}$ & $\begin{array}{l}\text { - Excédents d'azote : risque de pollution des eaux } \\
\text { de surface et des nappes (algues vertes, odeurs, } \\
\text { pollution de l'air) } \\
\text { - Dégâts sur les écosystèmes }\end{array}$ \\
\hline
\end{tabular}

L'emploi agricole représente $4 \%$ de l'emploi régional, un des niveaux les plus élevés en France (Agreste Bretagne 2016). Les industries agroalimentaires, implantées depuis longtemps sur le territoire, font travailler près de 68300 salariés en 2012, soit $40 \%$ de l'emploi industriel de la région. Au total, les emplois liés à l'agriculture, dont la part principale est liée à l'élevage, représentent 9,5\% des emplois totaux. Les travaux de Lorre et al (2015) indiquent qu'en moyenne pour l'élevage français, chaque emploi direct en élevage contribue à respectivement $1,1-0,8-6,1-1,9$ et 1,8 emplois indirects en filière lait, bovin viande, porc, volaille de chair et œuf. Les productions de volailles et surtout de porcs génèrent un nombre important d'emplois indirects en amont et surtout en aval de la production. Ceci constitue un atout pour la région, mais rend à l'inverse la compétitivité de ces filières très dépendante du coût du travail.

\section{5 / Mise en évidence de synergies ou compromis}

Les principaux impacts positifs et négatifs des systèmes de polyculturepolyélevage présents en Bretagne, avec une forte densité d'élevages, sont synthétisés sur la figure 3 et au tableau 3. Ils illustrent aussi les aspects réglementaires et les synergies ou antagonismes entre acteurs (Duru et al 2017, ce numéro).
Ces systèmes d'élevages se caractérisent par une production élevée par unité de surface et par unité de travail avec un recours important à des intrants, principalement pour l'alimentation des animaux, alors que la consommation d'engrais chimiques est relativement limitée du fait de la valorisation des déjections animales. Les services d'approvisionnement, en particulier la production de protéines animales pour l'alimentation humaine (couvrant un tiers de la consommation des français), constituent la principale fonction de ces systèmes.

Les impacts environnementaux locaux liés aux émissions de nitrate, de phosphore et d'ammoniac sont une limite importante de ces systèmes. Malgré des progrès significatifs en termes de réduction des flux de nitrate et la baisse des effectifs d'animaux depuis dix ans. À l'inverse, les impacts globaux en termes d'émission de GES par unité de produit sont particulièrement faibles.

$\mathrm{Au}$ niveau régional, l'élevage contribue significativement à l'activité économique et à l'emploi ce qui favorise son acceptabilité locale, même si ses nuisances (odeurs, bruit) peuvent également la pénaliser. Ceci constitue un atout pour le territoire mais peut aussi constituer une faiblesse si le coût du travail est plus élevé que dans les bassins de production concurrents.

\section{2 / Développement de la méthanisation dans les zones d'élevage en Allemagne}

\section{1 / Contexte}

a) Un développement rapide de la méthanisation depuis les années 2000, mais qui tend à plafonner

En Allemagne, la production de biogaz s'est fortement développée au cours de ces dix dernières années dans un contexte politique très favorable visant à la fois à l'accroissement de la production d'énergie renouvelable et la réduction des émissions de GES par les déjections animales (figure 4). Ce développement s'inscrit dans une volonté politique d'accroître la part des énergies renouvelables pour la production d'électricité à $40-45 \%$ en 2025 et $50-60 \%$ en 2035 , tout en réduisant les émissions de gaz à effet de serre de 50\%. En 2014, le nombre d'installations de biogaz atteignait 8700 pour une puissance électrique de près de $4000 \mathrm{MW}$ (Fachverband Biogas 2014), soit environ l'équivalent de quatre centrales nucléaires. À titre de comparaison, on comptait en 2012 seulement 300 méthaniseurs en France. La valorisation de l'énergie sous la forme d'électricité est très majoritaire. La chaleur produite par la cogénération est en fréquemment 
Figure 4. Évolution en Allemagne du contexte réglementaire et du nombre d'installations de biogaz et de la puissance électrique associée (Fachverband Biogas 2014).

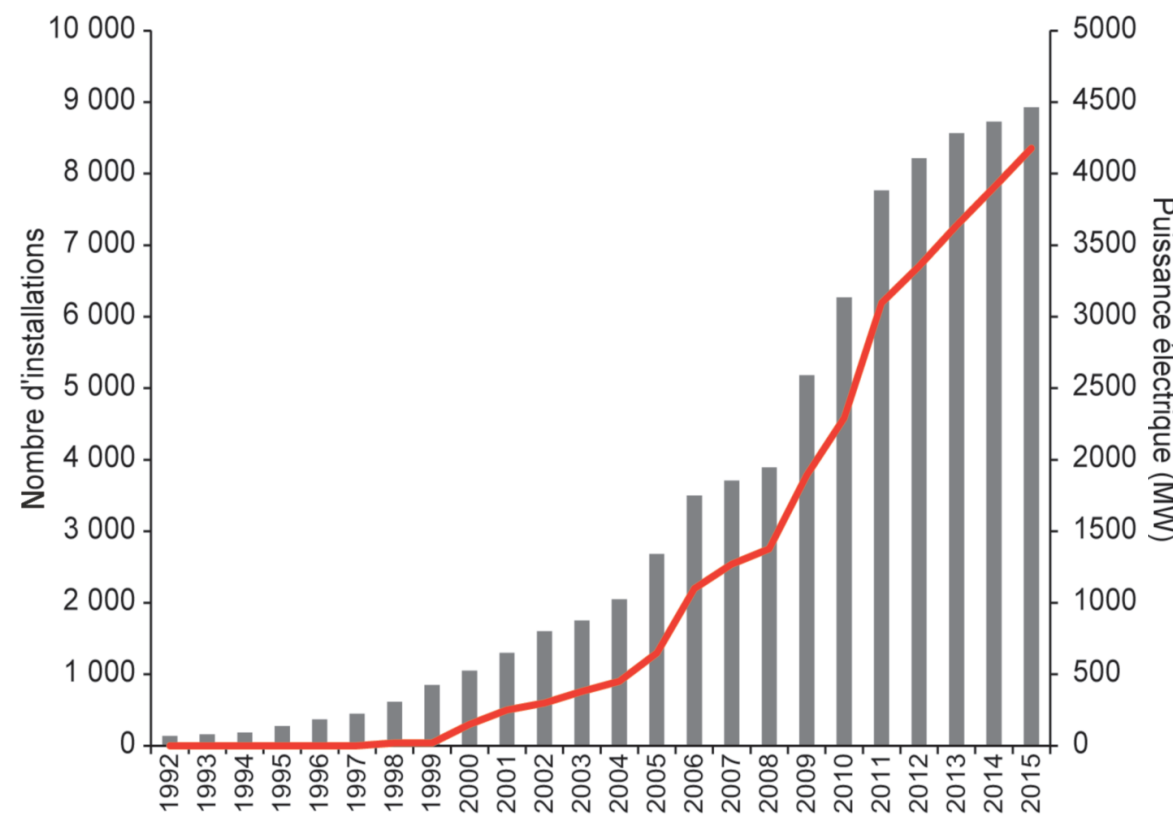

Nombre d'installations

— Puissance électrique (MW)

utilisée pour le chauffage domestique (Fachverband Biogas 2014). Le développement de la production de biogaz a été particulièrement rapide dans les années 2009 à 2011 avec plus de 1200 installations nouvelles par an; il se poursuit à un rythme moins élevé depuis 2013, avec seulement 300 nouvelles installations par an (figure 4). Cet important développement a été réalisé dans le cadre de quatre lois successives sur les énergies renouvelables. Le principe initial présent dans la loi de 2000 consistait à garantir un prix de reprise de l'électricité sur une période de 20 ans. Les lois suivantes ont ensuite rajouté différents bonus privilégiant certaines technologies et favorisant une accélération du développement à partir de 2009. À l'inverse, la loi de 2012 tend à être plus restrictive avec de nouvelles exigences d'efficacité énergétique et environnementale.

La majorité du biogaz est produit à partir de la co-digestion d'effluents animaux (bovins, porcin et volailles) en association avec des cultures et des résidus de culture (Fachverband Biogas 2014). On peut ainsi constater que la densité de digesteurs est liée à la densité de production laitière et porcine. La méthanisation reste donc généralement associée à l'élevage dont les effluents s'avèrent être des substrats particulièrement intéressants. Ils apportent l'ensemble des nutriments et micro-nutriments nécessaires au développement des microorganismes et, du fait de leur fort pouvoir tampon, ils permettent de stabiliser le $\mathrm{pH}$ du digesteur, ce qui est un atout majeur de stabilité du procédé (Béline et al 2010, Weiland 2013). Le digestat est
$\mathrm{Au}$ total, les effluents d'élevage représentaient en moyenne $45 \%$ des substrats méthanisés, les cultures énergétiques $46 \%$ et les bio-déchets $7 \%$, mais compte tenu de leur plus forte teneur en eau la contribution des effluents d'élevage à la production de biogaz est bien moindre que celle des cultures (Weiland 2013). Elle s'est néanmoins récemment accrue avec la mise en place d'une prime lorsqu'ils représentent plus de $10 \%$ du pouvoir méthanogène de la biomasse (Thiering et Bahrs 2011).

\section{b) La méthanisation dans les exploitations biologiques}

La méthanisation s'est également développée dans les exploitations biologiques dans lesquelles on comptait en 2012 près de 200 digesteurs (Gerlach et al 2013). Les principales spécificités de la production de biogas « bio » sont rapportées par (Gerlach et al 2013). Elle combine la production d'énergie renouvelable et l'agriculture biologique dans une approche intégrée. Le digesteur est utilisé à la fois pour la production d'énergie (chaleur, gaz ou électricité) et la fourniture et le recyclage d'éléments fertilisants pour les cultures. La biomasse utilisée est principalement issue de l'AB, de la transformation de produits issus de l'AB et de l'entretien de milieux naturel. Il s'agit surtout d'effluents d'élevage, de récoltes de cultures intermédiaires (cipan) et de cultures dédiées, généralement des légumineuses ou des prairies, de résidus agroalimentaires et de déchets de cuisine (sans OGM et sans métaux lourds). L'utilisation de cultures énergétiques est limitée afin d'éviter la compétition en surface pour la production alimentaire. Les digestats sont utilisés comme fertilisants sur l'exploitation, afin d'assurer le recyclage des éléments et accroître la fertilité des sols. Le digesteur est ainsi utilisé comme "outils" de production de fertilisants azotés "biologiques" permettant d'accroître la productivité de l'ensemble du système.

\section{2 / Services d'approvisionnement}

La production de biogaz contribue à la production d'énergie renouvelable principalement sous forme d'électricité, et dans une moindre mesure sous forme de gaz injecté dans les circuits de distribution et sous forme de chaleur (provenant de la cogénération d'électricité) utilisée principalement pour le chauffage domestique. En 2014, la production électrique allemande issue de la méthanisation s'élevait à 32,1 TWh (tableau 4), soit $21 \%$ de la production d'électricité renouvelable, l'électricité éolienne, photovoltaïque et hydroélectrique contribuant à respectivement 34,31 et $13 \%$ du total. L'électricité issue du biogaz correspond à 
l'équivalent de la consommation d'environ neuf millions de ménages (tableau 4).

Malgré le développement des cultures énergétiques pour la production de biogaz, la production de produits animaux par l'agriculture allemande s'est fortement accrue au cours de ces dernières années dans la plupart des filières. La production laitière a ainsi augmenté de $15 \%$ entre 2000 et 2014 (14\% depuis 2005 ), la production porcine de $40 \%$, la production d'œufs de $25 \%$ et la production de viande de volaille a doublé sur la même période. À l'inverse la production de viande bovine a diminué de $20 \%$ entre 2000 et 2014 (Destatis 2015). Au cours de cette période la production de céréales (hors ensilage de maïs) est restée relativement stable (autour de 45 millions de tonnes ; Destatis 2015), alors que les importations pour l'alimentation animale se sont accrues (de 4,8 à 8,7 millions de tonnes entre 2005 et 2012). Les exportations étant restées plus stables (autour de 10,5 millions de tonnes). Le solde positif des échanges de céréales a donc été réduit. Les importations de sources de protéines se sont également accrues principalement sous la forme de graine de colza $(+185 \%$, ces importations sont aussi en lien avec le développement de la production de diester) et dans une moindre mesure de tourteau et graines de soja $(+10 \%)$.

\section{3 / Services pour l'environnement}

La production d'électricité à partir de biogaz remplace celle issue de combustibles fossiles (charbon, lignite), ce qui conduit à une réduction des émissions de GES estimée à 20,8 millions de tonnes à l'échelle nationale en 2014 (Fachverband Biogas 2014, tableau 3.). Différentes études ont été consacrées à l'évaluation environnementale de la production d'électricité à partir de biogaz (Dressler et al 2012, Jury et al 2010). Elles indiquent une assez grande variabilité des résultats pour l'émission de gaz à effet de serre entre $-0,14$ et $+0,18 \mathrm{~kg}$ éq $\mathrm{CO}_{2}$ par $\mathrm{kWH}$ électrique. L'évaluation de l'épargne d'émission de $\mathrm{CO}_{2}$ dépend ensuite de l'hypothèse de substitution retenue, généralement la production d'électricité à partir du charbon ou du gaz. Les résultats de l'étude de Whiting et Azapagic (2014) indiquent ainsi que la production

Tableau 4. Les statistiques de production de biogaz en Allemagne (Fachverband Biogas 2014).

\begin{tabular}{|l|c|}
\hline & Année 2014 \\
\hline Nombre d'installations (dont avec injection de gaz) & $8726(178)$ \\
Puissance électrique installée (Mégawatt) & 3905 \\
Production d'électricité (Térawatt-heure) & 32,1 \\
Nombre de foyers-équivalent approvisionnés & 9200000 \\
Réduction de l'émission de $\mathrm{CO}_{2}$ (million de tonnes) & 20,8 \\
Turnover financier (milliards Euros) & 9,1 \\
Nombre d'emplois dans le secteur du biogaz & 45000 \\
\hline
\end{tabular}

d'électricité à partir de biogaz réduit plusieurs des impacts environnementaux, comparativement aux alternatives d'origine fossile. C'est le cas en particulier pour l'émission de GES (jusqu'à 50\% plus faible), l'épuisement des ressources abiotiques (qui sont préservées par le biogaz), l'écotoxicité aquatique ou terrestre et la toxicité humaine. Les effets sont cependant moins marqués en comparaison du gaz naturel que relativement aux autres sources d'énergie, charbon et lignite.

À l'inverse des bénéfices observés visà-vis des émissions de GES, les impacts potentiels d'eutrophisation et d'acidification sont fortement accrus du fait des émissions d'ammoniac et de la lixiviation de nitrate (multiplié par 20 et par 10 , respectivement), les combustibles fossiles n'ayant pratiquement pas d'impacts. L'utilisation de substrats plus énergétiques, comme l'ensilage de maïs, permet d'accroitre l'efficacité énergétique et de réduire les émissions de GES, mais s'accompagne généralement d'une augmentation des impacts acidification et d'eutrophisation. Il peut donc exister un «trade-off 》 entre production d'énergie renouvelable par méthanisation et protection de la ressource en eau (Svoboda et al 2013) et de la qualité de l'air. L'accroissement de la teneur en nitrate des eaux souterraines et de surface est ainsi à l'origine d'une mise en cause de l'Allemagne devant la Cour Européenne de Justice en 2016. La teneur en nitrate dépasse ainsi la valeur limite de $50 \mathrm{mg} / \mathrm{L}$ pour $30 \%$ des points de mesure en Allemagne et même pour plus de $50 \%$ des cas dans les länder Schleswig-Holstein et Basse-Saxe. Comparativement à l'ensilage de maïs, l'utilisation d'ensilage d'herbe conduit à une perte de nitrate tout en restant limitée par hectare lorsque les apports de fertilisants respectent les recommandations (Svoboda et al 2013). La production de biogaz par ha est toutefois inférieure avec l'ensilage d'herbe.

D'autres effets négatifs sur l'environnement du développement de la méthanisation sont avancés par certains auteurs. Gutzler et al (2015) ont simulé l'effet d'un scénario de développement des cultures énergétiques pour la méthanisation dans le Land de Brandebourg en comparaison inférieure par unité de méthane produite

avec les pratiques actuelles. Celui-ci s'accompagne d'un accroissement des surfaces de maïs dont la part passe de $29 \%$ à $49 \%$ de la SAU. Un tel scénario permet d'accroître la part d'énergie renouvelable du Land jusqu'à $41 \%$ et épargne l'émission de 3,5 millions de tonnes eq $\mathrm{CO}_{2}$ de GES. Par contre, il entraîne une perte de biodiversité, une détérioration de l'attractivité des paysages, un accroissement des risques d'érosion des sols et d'eutrophisation, en particulier dans les zones les plus fertiles.

Le développement de la production de biogaz pourrait également entraîner une conversion en culture des surfaces en herbe, et donc un moindre stockage du carbone dans les sols. À notre connaissance, cet aspect est peu connu de même que l'influence directe de la méthanisation sur la teneur en carbone des sols, qui semble dépendre des modalités de gestion des digestats et de la nature des substrats utilisés. Inversement, la méthanisation de la prairie permanente est parfois envisagée comme une alternative pour la maintenir dans des territoires où la déprise de l'élevage est marquée (Lebuhn et al 2014). Selon une étude conduite en Bavière, une déprise de près de 200000 ha de pâturage est ainsi envisagé d'ici 2020 avec des opportunités de développement de la production de biogaz à base d'herbe.

\section{4 / Impacts socio-économiques}

L'accroissement de la production d'énergie renouvelable en remplacement des énergies fossiles et nucléaire répond à une demande sociétale forte et à des choix politiques favorisant le développement du photovoltaïque, de l'éolien et de l'utilisation de la biomasse (EEG 2014). Ceci s'est traduit par des incitations financières forte, en particulier des garanties de prix de reprise de l'électricité ou du gaz (Weiland 2013). De plus, les consommateurs allemands expriment un fort consentement à payer pour des sources d'électricité moins polluantes (Longo et al 2008), ce qui contribue également à leur essor. Après avoir plusieurs fois évolué, le système de rémunération de base de l'électricité produite à partir du biogaz dépend de la puissance électrique et favorise les petits digesteurs. Il est complété par différentes primes, en fonction du type de substrat utilisé (en faveur des effluents d'élevage et des résidus d'entretien du paysage comparativement aux cultures énergétiques) et du mode de valorisation de l'énergie (en faveur de l'utilisation directe du gaz) (Weiland 2013). La réglementation actuelle est donc particulièrement favorable aux petits digesteurs $(<75 \mathrm{kWh})$ utilisant surtout des effluents d'élevage et peu d'ensilage de maïs et valorisant directement le biogaz. Ces nouvelles orientations 
visent à limiter le développement de biogaz à partir de culture énergétique, en particulier le maïs, qui commence à être remis en cause (Lebuhn et al 2014).

Selon les résultats de Reise et al (2012), les motivations des exploitants pour investir sont en grande partie liées au coût du capital, à une perception subjective des risques qu'ils prennent, et à la recherche d'une diversification de leur source de revenu Les considérations non monétaires comme la durabilité semblent jouer un rôle assez mineur. Les exploitants sont également sensibles aux incitations financières. La production d'éner-

Figure 5. Représentation graphique du cas d'étude "Allemagne du Nord » selon le formalisme de la grange.

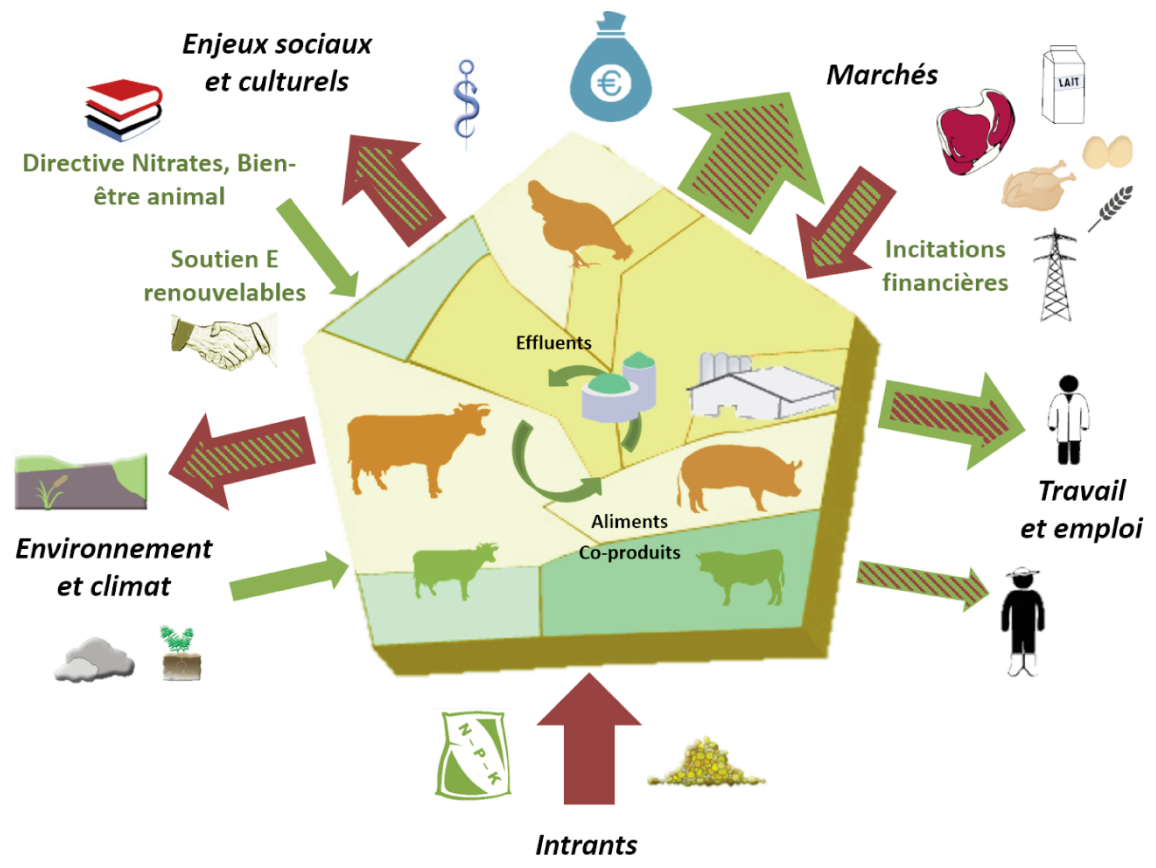

gie (solaire, photovoltaïque et biogaz) est la principale source de diversification des exploitations agricoles allemandes (49\%), suivie de la production de bois (22\%), la vente directe $(15 \%)$, le cheval et le tourisme (respectivement 13 et $9 \%$; Destatis 2015).

On estime à 378000 , le nombre d'emplois créés en Allemagne par le développement d'énergie renouvelable (Lebuhn et al 2014), dont environ 45000 dans l'industrie du biogaz (tableau 4). Toutefois, compte-tenu de l'évolution récente de la réglementation et des prix de reprise de l'électricité, en particulier pour les installations de grande taille (EEG 2014), la perspective de dépasser les 9000 installations est peu probable et des suppressions d'emplois sont en cours dans le secteur (Lebuhn et al 2014). L'activité économique liée au biogaz était estimée à environ 9 milliards d'Euros en 2014 (tableau 4). Après une croissance forte, elle tend à stagner voire à régresser ces dernières années (Lebuhn et al 2014).

\section{5 / Mise en évidence de quelques synergies ou compromis}

L'ensemble des impacts positifs ou négatifs des unités de méthanisation associées à des exploitations d'élevage en Allemagne sont représentés de manière synthétique à la figure 5 et au tableau 5 .

Tableau 5. Impacts positifs et négatifs associés aux territoires à forte densité d'élevages de l'Allemagne du Nord.

\begin{tabular}{|c|c|c|}
\hline $\begin{array}{l}\text { Interfaces } \\
\text { étudiées }\end{array}$ & Effets positifs & Effets négatifs \\
\hline Intrants & $\begin{array}{l}\text { - Utilisation des digestats comme fertilisants } \\
\text { - Contribution à la production d'énergie }\end{array}$ & $\begin{array}{l}\text { - Effets délocalisés (usage des terres, énergie, } \\
\text { pesticides) pour la production des aliments } \\
\text { concentrés } \\
\text { - Utilisation de pesticides et engrais }\end{array}$ \\
\hline $\begin{array}{l}\text { Travail } \\
\text { et emploi }\end{array}$ & $\begin{array}{l}\text { - Emplois directs créés par l'industrie } \\
\text { du biogaz }\end{array}$ & $\begin{array}{l}\text { - Stagnation récente des installations } \\
\text { de grande taille en raison de l'évolution } \\
\text { de la réglementation et du prix de reprise } \\
\text { de l'électricité }\end{array}$ \\
\hline Marchés & $\begin{array}{l}\text { - Prix de reprise de l'électricité élevé } \\
\text { - Diversification des ressources } \\
\text { de l'exploitation } \\
\text { - Production de masse à prix très compétitifs } \\
\text { - Incitations financières }\end{array}$ & $\begin{array}{l}\text { - Dépendance vis-à-vis de la conjoncture } \\
\text { internationale (prix des matières premières } \\
\text { et mise sur le marché) }\end{array}$ \\
\hline $\begin{array}{l}\text { Enjeux sociaux } \\
\text { et culturels }\end{array}$ & $\begin{array}{l}\text { - Soutien politique à la production d'énergie } \\
\text { renouvelable } \\
\text { - Demande sociale forte pour le remplacement } \\
\text { des énergies fossiles et du nucléaire } \\
\text { - Possible association avec l'agriculture } \\
\text { biologique }\end{array}$ & $\begin{array}{l}\text { - Remise en cause des cultures énergétiques } \\
\text { - Prise en compte des impacts } \\
\text { environnementaux } \\
\text { - Esthétique des paysages } \\
\text { - Accroissement du prix de la terre }\end{array}$ \\
\hline $\begin{array}{l}\text { Environnement } \\
\text { et climat }\end{array}$ & $\begin{array}{l}\text { - Valorisation des effluents d'élevage } \\
\text { avec de moindres émissions directes } \\
\text { et indirectes de GES } \\
\text { - Services intrants fournis par les prairies. }\end{array}$ & $\begin{array}{l}\text { - Impacts d'eutrophisation et d'acidification } \\
\text { accrus } \\
\text { - Conversion des prairies en culture } \\
\text { - Déstockage de carbone des sols } \\
\text { - Biodiversité }\end{array}$ \\
\hline
\end{tabular}


Ils illustrent également les aspects réglementaires et les synergies ou antagonismes entre acteurs. Les services d'approvisionnement en denrées alimentaires continuent à constituer la finalité principale du système de production. La production d'énergie par méthanisation est une voie de diversification pour les exploitations en leur garantissant un revenu complémentaire aux activités agricoles. Cette nouvelle activité s'est développée dans un contexte politique favorable répondant à une demande sociétale forte pour l'abandon du nucléaire et le développement des énergies renouvelables. Il n'y a pas eu d'antagonisme dans la croissance des activités d'élevage et de production d'énergie puisque toutes deux ont très fortement progressé ces quinze dernières années. Il peut même y avoir eu des synergies économiques. Le pays a accru son autosuffisance alimentaire et ses exportations de produits animaux tout en développant sa production d'énergie renouvelable.

Pour ce qui est des impacts environnementaux il peut, dans certaines conditions, exister un compromis entre d'un côté la production d'énergie renouvelable et la réduction des émissions de GES, et de l'autre, la protection de la ressource en eau, de la qualité de l'air et la préservation de la biodiversité. Ce risque est toutefois mieux pris en compte dans la dernière loi sur les énergies renouvelables. Le consentement à payer pour des énergies renouvelables est élevé en Allemagne, ce qui a favorisé leur essor au travers d'aides à l'investissement et des prix de reprise élevé de l'électricité. L'acceptabilité sociale de cette technologie a donc été relativement élevée, mais elle tend à se réduire en raison d'interrogations croissantes relatives aux possibles effets négatifs sur l'environnement et les prix agricoles, et au recours à des cultures énergétiques. À moyen terme se pose également la question du prolongement des contrats de reprise de l'électricité.

\section{3 / La Catalogne, un territoire marqué par un développe- ment récent et rapide de la production porcine dans un milieu où les surfaces agri- coles sont limitées}

\section{1 / Contexte}

a) Un développement rapide d'une production porcine spécialisée et intégrée

L'agriculture de la Catalogne, comme certaines autres régions Européennes est marquée par la prédominance de l'élevage, et plus particulièrement de la production porcine qui a doublé depuis 1980 pour atteindre 7 millions de porcs en 2014 (soit un cheptel très proche de celui de la Bretagne qui compte 7,8 millions de têtes). Il s'agit donc d'un développement particulièrement rapide, qui s'est progressivement étendu sur la province d'Aragon, et beaucoup plus récent que dans les autres bassins de production européen. Ce développement a été favorisé par un marché intérieur très porteur puis par les exportations en particulier vers la France.

Cet accroissement de la production a été associé à une réduction du nombre d'élevages et un fort accroissement de leur taille. Ceci s'explique à la fois par la disparition des petites exploitations et le développement des plus grandes avec des investissements importants et des choix technologiques standardisés en bâtiment, génétique et nutrition. La production a donc évolué vers un modèle d'élevages très spécialisés, de grande taille, avec un modèle contractuel d'intégration qui permet pour les éleveurs de développer l'activité à moindre risque et ainsi de mieux faire face aux fluctuations des prix et à la globalisation du marché européen. Dans cette organisation, l'éleveur est propriétaire des bâtiments et des équipements, gère les effluents et fournit le travail. La firme intégratrice qui reste propriétaire des animaux fournit les intrants et services à l'élevage et gère la commercialisation des animaux aux abatteurs. Dans certains cas (environ 25\% de la production), la firme intégratrice est impliquée dans l'abattage. Ces élevages disposent généralement de peu ou pas du tout de surface agricole. Les effluents sont épandus chez des tiers, assez souvent dans le cadre de plan d'épandage collectifs (voir ci-après) et les aliments proviennent de l'extérieur.

b) La Catalogne, une région avec une prédominance des espaces naturels et peu de surface agricole

La Catalogne se caractérise par une forte prédominance de l'occupation de l'espace par la forêt et les taillis qui représentent environ 64\% de la superficie du territoire ainsi que par un faible indice de diversité paysager («Shannon evenness index »). La SAU représente seulement un tiers de la superficie environ soit une valeur bien plus faible que dans la plupart des autres régions (par exemple $68 \%$ dans le Grand Ouest de la France). Cette faible proportion de la surface agricole peut à la fois avoir des effets positifs et négatifs. L'importance des espaces naturels contribue à un effet de dilution des émissions gazeuses et des émissions vers l'eau. Ceci explique qu'à l'échelle européenne, la Catalogne n'apparait pas véritablement comme une " zone sensible » pour les nitrates ou l'ammoniac même si plusieurs cantons sont concernés. À l'inverse, la faible disponibilité des surfaces cultivées rend plus difficile la bonne valorisation des effluents comme fertilisant, d'autant plus que la répartition de l'élevage porcin à l'échelle régionale n'est pas homogène. Six des 42 cantons hébergent près de $60 \%$ de la totalité du cheptel.

c) Un renversement de la politique des énergies renouvelable en Espagne mettant un frein à la méthanisation

Dans les années 1990, le gouvernement espagnol, à l'instar de son homologue allemand, a mis en place une législation favorisant le développement des énergies renouvelables. Les incitations financières ont alors favorisé l'essor de la méthanisation. En parallèle, le gouvernement catalan a instauré un plan pour développer la méthanisation et traiter ainsi de 1,3 million de $\mathrm{m}^{3}$ de lisier. Mais ces dernières années, la crise économique espagnole a remis en cause les incitations financières aux énergies renouvelables et la majorité des installations (40 environ) ont fermé par manque de rentabilité, pour l'équivalent de $800000 \mathrm{~m}^{3}$ d'effluents.

\section{2 / Services d'approvisionnement}

L'importance du secteur agroalimentaire de la Catalogne est très significative aussi bien au niveau espagnol qu'au niveau européen. L'observatoire européen des clusters (2016) place la Catalogne parmi les premières régions pour les produits alimentaires, compte tenu de l'importance du secteur et du nombre d'emplois générés. L'industrie agro-alimentaire représente $20,7 \%$ des ventes nettes totales de l'industrie catalane. En 2012, le chiffre d'affaires agroalimentaire catalan atteignait 20 milliards $€$ et, avec 75000 emplois, la Catalogne représentait 21,3\% de l'ensemble des emplois de l'industrie agroalimentaire espagnole. Cette situation est en grande partie liée au secteur de l'élevage. La Catalogne est devenue non seulement un grand producteur mais aussi un grand transformateur de produits carnés. L'industrie de transformation de la viande a un des taux de profitabilité (poids de l'excédent brute d'exploitation dans le chiffre d'affaires) les plus élevés d'Europe. Les filières de production situées à proximité de l'abattage et de la transformation ont su accroître leur productivité et ont profité du développement des infrastructures de transport pour l'approvisionnement du marché national et pour l'exportation. Le poids de l'Espagne dans les exportations européennes de produits porcins a quasi doublé entre 2000 et 2014 , passant de 7 à $13 \%$. 


\section{3 / Services pour l'environnement}

a) Une approche intégrée des problèmes environnementaux à l'échelle des territoires

Les problèmes environnementaux liés à l'utilisation de l'azote étant principalement causées par une mauvaise gestion plutôt qu'à un excès global, le ministère catalan de l'Agriculture, de l'Élevage, des Pêches et de l'Alimentation a lancé un programme régional axé sur l'amélioration des pratiques de fertilisation. L'initiative a débuté en 2001 avec un programme pilote dans le nord-est de la Catalogne qui a été rapidement étendu à d'autres régions. Il est actuellement composé de six programmes en cours dans six grandes régions du pays, chacun d'entre eux étant adapté aux caractéristiques de chaque zone géographique : type de sol, climat, type de cultures et pratiques agricoles. Ces plans associent des éleveurs qui disposent de peu ou pas de surface d'épandage et des agriculteurs producteurs de culture ayant des besoins de fertilisation. L'ensemble de la gestion des effluents (plans de fertilisation, transport et gestion des épandages, gestion administrative...) est assurée par des structures collectives à l'interface entre les éleveurs et les agriculteurs.

L'objectif principal de ces programmes est l'amélioration de la fertilisation organique et la réduction de la fertilisation minérale. Ceci passe par la mise en œuvre chaque année de plusieurs expérimentations de terrain couvrant les différentes cultures dans toute la Catalogne. Des conseils personnalisés sont donnés aux agriculteurs sélectionnés avant chaque période de fertilisation. Près de 400 agriculteurs reçoivent actuellement des conseils de techniciens spécialisés dans plusieurs cultures comme l'orge, le blé, le maïs, le sorgho, le colza, les cultures fourragères, d'oliviers ou de riz.

b) Le développement de plans collectifs de traitement/valorisation des effluents

La gestion collective des effluents a démarré en 2002, le premier plan collectif ayant été approuvé à cette époque par le Conseil de l'agriculture dans le cadre de la directive Nitrate. Depuis, quarante autres plans collectifs ont été mis en œuvre, impliquant 2200 exploitations. Une des raisons de l'instauration de ces plans de gestion a été la tentative de construire des usines centralisées de traitement des effluents, pour accéder à des économies d'échelle. L'objectif était de traiter une partie des effluents et d'améliorer leur gestion dans les zones à forte densité d'élevage. Actuellement, environ $20 \%$ des effluents, principalement de porcs et de bovin, sont gérés de cette manière. Les modalités mises en œuvre sont diversifiées comme par exemple :

i) Juncosa. Installation de compostage de fumiers de volailles et de bovins dans une zone dense et exportation à grande distance vers des zones déficitaires ;

ii) les Garrigues. Installation collective de méthanisation et de séchage des effluents pour la production d'un produit déshydraté facilement transportable et valorisable ;

iii) vic, Torelló et Ivars. Cooperatives assurant la fourniture d'intrants, d'équipement et de services visant à mieux gérer les effluents (matériels d'épandage innovants, transport à longue distance) ou à réduire les flux (techniques innovantes d'alimentation);

iv) artesa de Segre et Linyola. Fourniture de services administratifs et de gestion. Fourniture d'aliments à taux réduits en protéines.

Pour l'avenir, ces organisations collectives seront également mobilisées par le gouvernement pour la mise en place de nouvelles approches innovantes comme i) la séparation de phase à la ferme ; ii) le transport longue distance des solides pour réduire la charge en phosphore ; iii) de nouveaux outils pour améliorer le gestion des effluents (prédiction de la valeur fertilisante, systèmes « intelligents » d'épandage, utilisation de GPS...) ainsi que $i v$ ) la réduction des flux de $\mathrm{N}$ et $\mathrm{P}$ par l'alimentation.

\section{c) La prise en compte du phosphore, une préoccupation plus récente}

Les analyses réalisées ces dernières années indiquent qu'un pourcentage élevé des sols agricoles de la Catalogne (plus de $50 \%$ des parcelles échantillonnées dans le projet Futur-Agrari ${ }^{1}$ ) présente des teneurs élevées en P. Cette accumulation du P dans les couches superficielles résulte de l'épandage continu d'effluents riches en $\mathrm{P}$ sur des sols calcaires qui retiennent le phosphore du fait d'un pH élevé. En accord avec la Commission Européenne et la plateforme européenne sur le phosphore, le Ministère de l'Agriculture s'inquiète de cette évolution et plaide pour le développement de la séparation de phase. Cette technologie permet en effet de concentrer le P dans la phase solide et de l'exporter ainsi plus facilement, la phase liquide étant épandue localement. Le gouvernement Catalan s'inscrit dans cette perspective en favorisant le développement de cette technologie, une meilleure connaissance de la situation actuelle et des plans de fertilisation mieux adaptés.

\section{4 / Impacts socio-économiques}

\section{a) Production d'énergie renouvelable}

Le volume actuel d'effluents d'élevage traités par méthanisation n'est plus que d'environ $280000 \mathrm{~m}^{3}$, correspondant à douze petites installations, un seul méthaniseur collectif restant en fonctionnement. L'objectif visé initialement n'a donc pas été atteint en raison de la faible rentabilité des projets suite à la suppression des aides à la reprise de l'énergie, mais également pour des raisons administratives et la difficulté à valoriser les digestats. Cependant, on observe ces dernières années, un intérêt nouveau pour cette technologie, à l'échelle des exploitations individuelles.

b) Meilleure valorisation des effluents et réduction de l'utilisation d'engrais : un intérêt économique et environnemental

La mise en œuvre de la directive sur les nitrates dans les fermes catalanes a été lente, mais les premiers résultats se font sentir. Toutefois, selon le ministère de l'agriculture, la réduction significative des concentrations en nitrate nécessitera quelques années. Traditionnellement, les effluents d'élevage étaient considérés comme des déchets sans réelle valeur, les agriculteurs continuant à utiliser des engrais chimiques (NPK) en plus des effluents organiques. La consommation d'engrais minéraux a augmenté en Catalogne depuis les années 1950 pour atteindre un pic entre 1998 et 2004. Depuis cette période, la meilleure valorisation des engrais organiques (100 000 tonnes d'azote 32000 tonnes de phosphore) a permis de réduire l'utilisation des engrais et des coûts de fertilisation. Ceci reflète une évolution des techniques mais aussi de la perception même des effluents par les agriculteurs céréaliers. Le rôle du support technique apporté par le Ministère de l'Agriculture auprès de 400 céréaliers a été particulièrement important. De même, en relation avec l'évolution de la réglementation, la recherche a produit de nombreuses informations particulièrement utiles en pratique. Les agriculteurs font preuve de beaucoup d'intérêt pour l'application de nouvelles technologies permettant d'améliorer la fertilisation minérale et organique (télédétection, logiciels de pilotage de la fertilisation, équi- 
Figure 6. Représentation graphique du cas d'étude « Catalogne » selon le formalisme de la grange.

Pour la signification des pictogrammes et des flèches se reporter à la légende de la figure 3.

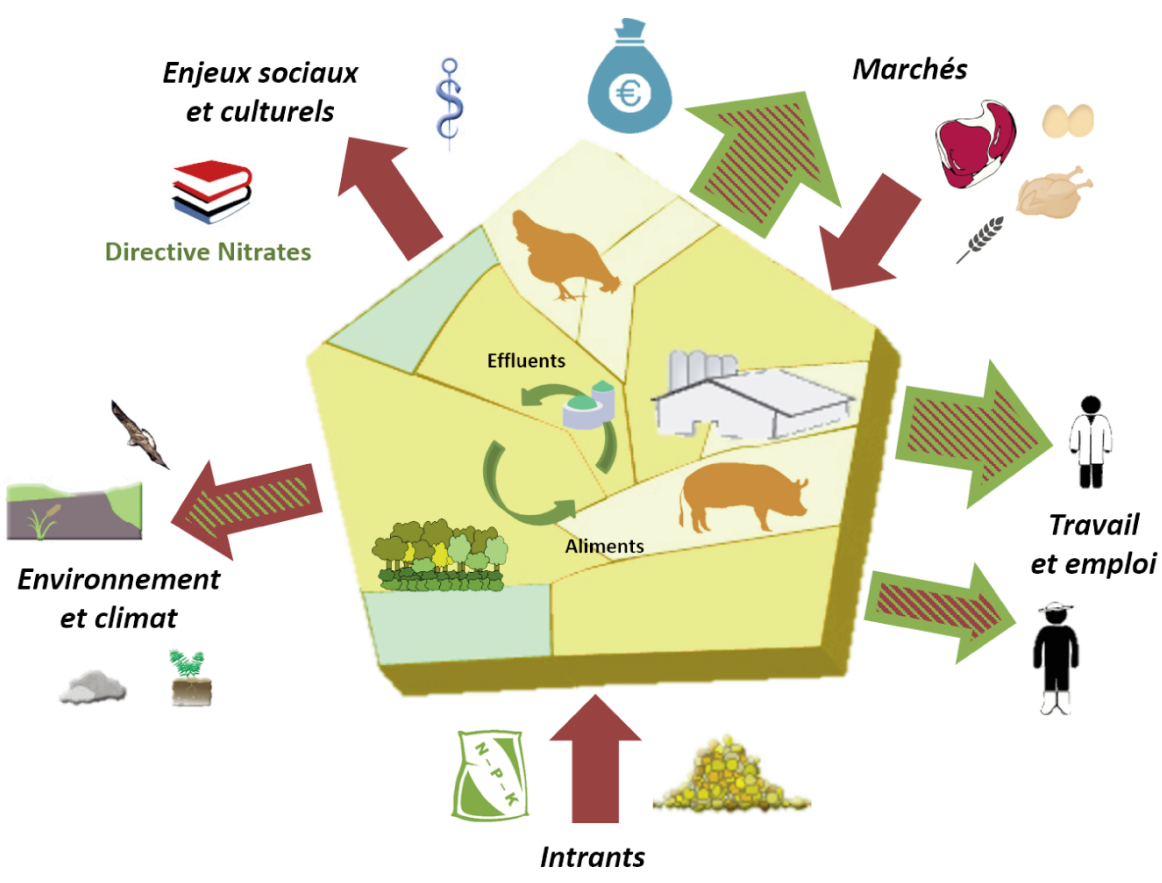

pements d'épandage de précision) et par là-même leur rentabilité. Ces perspectives s'inscrivent également dans les stratégies du gouvernement catalan pour améliorer l'utilisation des effluents dans les prochaines années.

\section{5 / Mise en évidence de quelques synergies ou compromis}

Les principaux impacts positifs et négatifs des systèmes d'élevages de Catalogne sont récapitulés de manière synthétique à la figure 6 et au tableau 6 qui illustrent également quelques synergies ou antagonismes entre acteurs. L'élevage de la Catalogne se caractérise par une croissance importante et récente avec une prédominance de la production de porcs dans des filières intégrées et la coexistence d'exploitations d'élevage de grande taille, généralement sans surface agricole avec des exploitations céréalières sans élevage. Cette organisation, s'est montrée particulièrement efficace pour maîtriser les coûts de production et s'adapter à la demande du marché national et européen. L'agriculture et l'agroalimentaire contribuent ainsi très significativement à l'économie aux emplois de la région.

Tableau 6. Impacts positifs et négatifs associés aux territoires d'élevage de Catalogne.

\begin{tabular}{|c|c|c|}
\hline $\begin{array}{l}\text { Interfaces } \\
\text { étudiées }\end{array}$ & Effets positifs & Effets négatifs \\
\hline Intrants & $\begin{array}{l}\text { - Recyclage des effluents organiques comme } \\
\text { fertilisants }\end{array}$ & $\begin{array}{l}\text { - Effets délocalisés (usage des terres, énergie, } \\
\text { pesticides) pour la production des aliments } \\
\text { concentrés } \\
\text { - Pesticides et engrais pour les cultures, } \\
\text { équipements }\end{array}$ \\
\hline $\begin{array}{l}\text { Travail } \\
\text { et emploi }\end{array}$ & $\begin{array}{l}\text { - Modèle contractuel d'intégration qui réduit } \\
\text { les risques } \\
\text { - Nombreux emplois dans les élevages } \\
\text { et les filières agroalimentaires }\end{array}$ & $\begin{array}{l}\text { - Réduction du nombre d'élevages, fort } \\
\text { accroissement de leur taille }\end{array}$ \\
\hline Marchés & $\begin{array}{l}\text { - Marché intérieur et à l'export porteur pour le } \\
\text { porc, maitrise du coût de production } \\
\text { - Forte présence sur le marché de la charcuterie } \\
\text { sèche } \\
\text { - Innovations technologiques en bâtiments, } \\
\text { génétique, nutrition }\end{array}$ & $\begin{array}{l}\text { - Dépendance vis-à-vis de la conjoncture } \\
\text { internationale (prix des matières premières } \\
\text { et mise sur le marché) }\end{array}$ \\
\hline $\begin{array}{l}\text { Enjeux sociaux } \\
\text { et culturels }\end{array}$ & - Acceptabilité locale liée à l'emploi & $\begin{array}{l}\text { - Risque accrus pour la santé animale du fait } \\
\text { des densités élevées } \\
\text { - Acceptabilité de l'élevage : bien-être animal, } \\
\text { nuisances olfactives, pollutions }\end{array}$ \\
\hline $\begin{array}{l}\text { Environnement } \\
\text { et climat }\end{array}$ & $\begin{array}{l}\text { - Faibles émissions directes et indirectes } \\
\text { de GES par kg de produit } \\
\text { - Dilution des émissions vers l'air et l'eau } \\
\text { par les espaces naturels }\end{array}$ & $\begin{array}{l}\text { - Accumulation de P dans les sols } \\
\text { - Mauvaises gestion et valorisation des effluents } \\
\text { car peu de surfaces pour l'épandage }\end{array}$ \\
\hline
\end{tabular}


L'élevage catalan s'inscrit sur un territoire où les zones naturelles occupent la majorité des surfaces, contribuant ainsi à diluer les impacts sur l'environnement mais réduisant aussi les surfaces disponibles pour l'épandage et rendant leur valorisation plus difficile. La prise en compte des réglementations environnementales est largement basée sur la volonté de mieux valoriser les effluents comme fertilisant, en favorisant les transferts entre éleveur et céréaliers dans le cadre de plan d'épandage collectifs, parfois en relation avec le développement d'installations de traitement.

\section{4 / En conclusion : quels leviers d'action pour les territoires à haute densité animale ?}

La présentation de la situation actuelle des bouquets de services dans les territoires « à haute densité animale » permet d'identifier un certain nombre de menaces qui pèsent sur les systèmes d'élevage de ces territoires :

i) La première est liée aux impacts négatifs sur l'environnement résultant de la concentration des élevages. Malgré de nettes améliorations et des options nouvelles proposées pour continuer à limiter les impacts, les territoires à haute densité animale sont toujours remis en cause par une frange croissante de la société, et sont encadrés par différentes politiques publiques compte-tenu de leurs impacts négatifs sur l'environnement. Cependant, comme illustré ci-dessus, haute densité animale ne va pas forcément de pair avec une faible diversité paysagère, ni avec des émissions de GES élevées par unité de produits ;

ii) ces systèmes font aussi face à un questionnement du modèle socio-économique dans lequel ils s'inscrivent avec des interrogations autour de la faible

Tableau 7. Principaux leviers d'action proposés pour les territoires « à haute densité animale ».

\begin{tabular}{|c|c|c|}
\hline $\begin{array}{l}\text { Leviers } \\
\text { d'action }\end{array}$ & Voie 1 : Recherche accrue d'efficience & $\begin{array}{c}\text { Voie } 2 \text { : Valorisation des services } \\
\text { écosystémiques }\end{array}$ \\
\hline $\begin{array}{l}\text { Échelle } \\
\text { troupeau, } \\
\text { exploitation }\end{array}$ & $\begin{array}{l}\text { Accroître l'efficience du système dominant } \\
\text { par l'agriculture de précision } \\
\text { Améliorer l'efficacité alimentaire des animaux : } \\
\text { - Alimentation de précision (multi-phases) } \\
\text { - Réduction de la teneur en protéines de la ration } \\
\text { (utilisation d'acides aminés de "synthèse") } \\
\text { Améliorer la gestion des effluents : } \\
\text { - Traiter les effluents par filtrage, séparation de } \\
\text { phase, compostage, séchage, etc } \\
\text { Améliorer le logement des animaux : } \\
\text { - Augmenter les surfaces par animal } \\
\text { - Enrichir le milieu de vie (litières...) } \\
\text { - Favoriser l'utilisation de bâtiments économes } \\
\text { en énergie ou rénover les anciens }\end{array}$ & $\begin{array}{l}\text { Favoriser l'autonomie alimentaire des } \\
\text { troupeaux en mettant la densité de l'élevage en } \\
\text { adéquation avec les potentialités du milieu } \\
\text { Limiter l'impact environnemental des systèmes } \\
\text { d'élevage en recréant du lien au sol : } \\
\text { - Diversifier les rotations et assolements pour } \\
\text { favoriser l'autonomie alimentaire des troupeaux } \\
\text { - Augmenter le recours au pâturage dans } \\
\text { l'alimentation pour les ruminants (en particulier en } \\
\text { développant les couverts de légumineuses) } \\
\text { - Limiter les concentrés dans les rations } \\
\text { Gérer la santé animale de manière intégrée : } \\
\text { - Limiter l'utilisation de médicaments par une } \\
\text { amélioration des pratiques (via logement, } \\
\text { alimentation...) } \\
\text { Limiter l'emprise des systèmes herbagers sur } \\
\text { les écosystèmes : } \\
\text { - Rechercher un seuil de chargement adapté au } \\
\text { compromis entre production et environnement } \\
\text { - Maintenir les prairies permanentes irremplaçables } \\
\text { pour leur rôle de maintien de la biodiversité, parfois } \\
\text { faire du sur-semis pour recréer des prairies } \\
\text { multifonctionnelles } \\
\text { - Préserver les infrastructures agroécologiques } \\
\text { (refuge de biodiversité...) }\end{array}$ \\
\hline $\begin{array}{l}\text { Échelle } \\
\text { territoire }\end{array}$ & $\begin{array}{l}\text { Recréer du lien au sol pour les animaux } \\
\text { Améliorer l'efficience du système dominant : } \\
\text { - Améliorer le métabolisme territorial par des } \\
\text { échanges intra-territoires (fertilisants, aliments) } \\
\text { - Développer des marques régionales pour } \\
\text { relocaliser la production et l'identifier } \\
\text { Favoriser l'économie circulaire : } \\
\text { - Développer les démarches collectives de gestion } \\
\text { des effluents (projets territoriaux de méthanisation, } \\
\text { plans d'épandage collectifs, banques de lisiers...) } \\
\text { - Recycler les déchets locaux dans l'alimentation } \\
\text { animale (drêches, pulpes, coproduits...) } \\
\text { - Instaurer des consultations collectives et une } \\
\text { gouvernance territoriale }\end{array}$ & $\begin{array}{l}\text { Développer et promouvoir des systèmes } \\
\text { alternatifs autonomes et/ou avec une image } \\
\text { de qualité } \\
\text { Renforcer les organisations de productions } \\
\text { locales promouvant des systèmes d'élevage } \\
\text { de qualité et liés au sol } \\
\text { Promouvoir les systèmes économes en intrants } \\
\text { liés au sol (Réseau Agriculture Durable...) } \\
\text { Favoriser l'autonomie de décision et le maintien } \\
\text { d'exploitations de petite taille, transmissibles }\end{array}$ \\
\hline
\end{tabular}


rémunération des éleveurs et des prix bas et/ou fortement fluctuants du marché pour les produits animaux ;

iii) enfin, un militantisme de la cause animale de plus en plus visible remet clairement en cause ces systèmes, en raison de leur atteinte au bien-être animal. Ceci a conduit à la mise en place de réglementations sur le bien-être animal qui ont contribué à l'évolution des modes d'élevage pour toutes les espèces.

Pour arbitrer les compromis entre services liés à ces menaces, il existe différents leviers applicables aux territoires à haute densité animale. Ces leviers s'inscrivent dans les deux voies de modernisation écologique décrites par Duru et al (2015) avec pour chacune d'entre elles des leviers à l'échelle des troupeaux et des exploitations d'élevage, et des leviers à l'échelle des territoires (tableau 7).

La première voie de modernisation écologique des systèmes passe en général par une recherche d'efficience alimentaire accrue. Dans une telle logique, on cherche à optimiser l'alimentation des animaux (entre autres par l'élevage de précision) de manière à valoriser leur potentiel génétique et permettre une croissance rapide ; ceci réduit simultanément les intrants nécessaires à la production et les impacts environnementaux liés aux déjections des animaux, et comptetenu de la forte contribution de l'alimentation aux charges opérationnelles des exploitations, ceci améliore également leur bilan économique. Améliorer le logement des animaux afin de leur offrir plus de surface et un environnement plus diversifié, par exemple en utilisant des litières, améliore leur bien-être et peut aussi contribuer à réduire les impacts locaux (odeurs, eutrophisation) (Garcia-
Launay et al 2014). En revanche, chez le porc, ceci peut s'accompagner d'un accroissement des émissions de gaz à effet $\left(\mathrm{N}_{2} \mathrm{O}\right)$ et d'une dégradation de la qualité des carcasses qui sont plus grasses (Lebret et al 2006). L'amélioration de l'efficience passe aussi par un meilleur recyclage des effluents d'élevage comme fertilisants et/ou leur utilisation pour la production d'énergie. Ceci peut être réalisé à la fois au niveau de l'exploitation, par exemple en limitant les émissions gazeuses dans le bâtiment ou pendant le stockage ou différentes échelles territoriales plus large, en favorisant les échanges entre exploitations ou en développant des démarche collectives de traitement et/ou de valorisation des effluents (Loyon et al 2016). Ces différentes démarches sont possibles grâce à différentes innovations technologiques qui permettent par exemple de séparer et/ou sécher les effluents afin de produire des engrais organiques facilement transférables vers des zones à faible densité animale. Ces stratégies s'inscrivent plus généralement dans des démarches d'économie circulaire qui concernent également l'approvisionnement en aliment, par exemple en favorisant des assolements mieux adaptés aux besoins des animaux, des échanges céréales-effluents, de nouvelles formes d'épandage ou une meilleure valorisation des différent coproduits disponibles sur le territoire (Lopez-Ridaura et al 2009, zu Ermgassen et al 2016).

La seconde voie de modernisation écologique mise sur l'augmentation de la fourniture de services écosystémiques utilisés comme support de la production. Ceci implique de recréer du lien au sol en favorisant l'autonomie alimentaire des troupeaux via une réduction de la densité des exploitations d'élevage afin de la mettre en adéquation avec les potentialités du milieu. L'usage des intrants de synthèse est alors réduit, mais aussi les niveaux de production et les pollutions. La marge brute de ces exploitations peut en revanche augmenter dès lors que la baisse de production est limitée, par exemple lorsqu'on optimise la conduite du pâturage (Dumont et al 2013). Pour les ruminants, cette voie passe ainsi le plus souvent par l'augmentation des surfaces en herbe. L'introduction des légumineuses dans les rotations, malgré les limites que peuvent poser les réglementations sur l'épandage des effluents, peut jouer un rôle important pour accroître les niveaux de production tout en améliorant la qualité des sols et en limitant les niveaux de fertilisation minérale. Dans un scénario " gagnant-gagnant », la préservation des prairies permet à la fois de nourrir les animaux à faible coût, de diversifier les paysages et de tamponner les risques de fuite d'azote vers le milieu (Peyraud et al 2012). Ceci peut se faire au niveau des exploitations mais a aussi un réel impact au niveau du territoire en raisonnant l'agencement des prairies et des infrastructures paysagères telles que les haies (Groot et al 2010). Pour les monogastriques, une voie prometteuse consiste en la mise en place de labels de qualité qui cherchent également à valoriser une image de bien-être animal (porcs élevés sur paille, volailles sur parcours). Certaines démarches existent déjà, surtout pour les volailles, mais elles pourraient se développer plus largement. Enfin, pour toutes les productions, une démarche de gestion intégrée de la santé des animaux (à l'échelle des exploitations mais aussi des territoires) permettrait de limiter l'usage des médicaments et de réduire les risques sanitaires par une amélioration de l'ensemble des pratiques (Dumont et al 2013).

\section{Références}

Agreste Bretagne, 2014. Tableaux de l'Agriculture Bretonne, 170p. http://agreste.agriculture. gouv.fr/IMG/pdf/R5315A07.pdf

Agreste Bretagne, 2016. Tableaux de 1'Agriculture Bretonne 2015, 93p. http://agreste. agriculture.gouv.fr/IMG/pdf/R5316A02.pdf

Béline F., Dabert P., Peu P., Girault R., 2010. La méthanisation des effluents d'élevage en France et en Europe : principe, état des lieux et perspectives. Fourrages, 203, 155-161.

Chatellier V., Magdelaine P., Trégaro Y., 2015. La compétitivité de la filière volaille de chair française : entre doutes et espoirs. INRA Prod. Anim., 28, 411-428.

Comité Régional Porcin, 2013. Le porc en Bretagne, chiffres clés 2013 : Comité Régional Porcin, 32p.

Destatis, 2015. Statistisches Bundesamt Wiesbaden. www.destatis.de/DE/ZahlenFakten/Wirtschafts
bereiche/LandForstwirtschaftFischerei/Land Forstwirtschaft.html (accessed 30.11.15)

Draaf Bretagne, 2013. L'élevage en Bretagne, hier, aujourd'hui et demain, $36 \mathrm{p}$.

Dreal Bretagne, 2014. L'eau en Bretagne, bilan annuel 2013, 47p

Dressler D., Loewen A., Nelles M., 2012. Life cycle assessment of the supply and use of bioenergy: impact of regional factors on biogas production. Int. J. Life Cycle Assess., 17, 11041115.

Dumont B., Fortun-Lamothe L., Jouven M., Thomas M., Tichit M., 2013. Prospects from agroecology and industrial ecology for animal production in the $21^{\text {st }}$ century. Animal, 7, 10281043.

Duru M., Moraine M., Therond O., 2015. An analytical framework for structuring analysis and design of sustainable ruminant livestock systems. Anim. Frontiers, 5, 6-13.

Duru M., Donnars C., Ryschawy J., Therond O., Dumont B., 2017. La « grange »: un cadre conceptuel pour appréhender les bouquets de services rendus par l'élevage dans les territoires. In : Numéro spécial, L'élevage en Europe : une diversité de services et d'impacts. Dumont B. (Éd). INRA Prod. Anim., 30, 273-284.

EEG, 2014. Erneuerbare-Energien-Gesetz. Gesetz für den Ausbau erneuerbarer Energien. http://www.gesetze-im-internet.de/eeg 2014/ (accessed 30.11.15).

Fachverband Biogas, 2014. http://biogas.org/ edcom/webfvb.nsf/id/DE Branchenzahlen/\$fil e/15-11-19 Biogasindustryfigures 20142015 english.pdf (accessed 30.11.15)

Gaigné C., 2012. Organisation des filières animales et environnement. Vingt ans après la directive nitrates. INRA Prod. Anim., 25, 233-244. 
Gaigné C., Letort E., 2017. Co-localisation des différentes productions animales en Europe : l'exception française ? INRA Prod. Anim., 30, 219-228

Gaigné C., Le Gallo J., Larue S., Schmitt B., 2012. Does regulation of manure land application work against agglomeration economies? Theory and evidence from the french hog sector. Am. J. Agr. Econ., 94, 116-132.

Garcia-Launay F., van der Werf H.M.G., Nguyen T.T.H., Le Tutour L., Dourmad J.Y., 2014. Evaluation of the environmental implications of the incorporation of feed-use amino acids in pig production using Life Cycle Assessment. Livest. Sci., 161, 158-175.

Gerlach F., Grieb B., Zerger U., 2013. Sustainable biogas production. A handbook for organic farmers. Frankfurt am Main, Germany: FiBL Projekte GmbH, 51p. http://www.sustaingas.eu/ handbook.html

Gibbs H.K., Rausch L., Munger J., Schelly I., Morton D.C., Noojipady P., Soares-Filho B., Barreto P., Micol L., Walker N.F., 2015. Brazil's Soy Moratorium. Sci., 347, 377-378.

Groot J.C.J., Jellema A., Rossing W.A.H., 2010. Designing a hedgerow network in a multifunctional agricultural landscape: Balancing trade-offs among ecological quality, landscape character and implementation costs. Europ. J. Agron., 32, 112-119.

Gutzler C., Helming K., Balla D., Dannowski R., Deumlich D., Glemnitz M., Knierim A., Mirschel W., Nendel C., Paul C., Sieber S., Stachow U., Starick A., Wieland R., Wurbs A., Zander P., 2015. Agricultural land use changes - a scenario-based sustainability impact assessment for Brandenburg, Germany. Ecol. Indicators, 48, 505-517.

Jury C., Benetto E., Koster D., Schmitt B., Welfring J., 2010. Life Cycle Assessment of biogas production by monofermentation of energy crops and injection into the natural gas grid. Biom. Bioenergy, 34, 54-66.

Lebret B., Meunier-Salaun M.C., Foury A., Mormede P., Dransfield E., Dourmad J.Y., 2006. Influence of rearing conditions on performance, behavioral, and physiological responses of pigs to preslaughter handling, carcass traits, and meat quality. J. Anim. Sci., 84, 2436-2447.

Lebuhn M., Munk B., Effenberger M., 2014. Agricultural biogas production in Germany from practice to microbiology basics. Energy Sustain. Soc., 4, 1-21.

Le Goffe P., 2008. La politique de l'eau : approche économique et application à la pollution des élevages. INRA Prod. Anim., 21, 419-426.
Longo A., Markandya A., Petrucci M., 2008 The internalization of externalities in the production of electricity: Willingness to pay for the attributes of a policy for renewable energy. Ecol. Econ., 67, 140-152.

Lopez-Ridaura S., van der Werf H., Paillat J.M., Le Bris B., 2009. Environmental evaluation of transfer and treatment of excess pig slurry by life cycle assessment. J. Environ. Manage., 90, 1296-1304.

Lorre G., Lang A., Dupraz P., Perrot P., Tregaro Y., Rosner P.M., 2015. Les emplois liés à l'élevage dans le Grand Ouest: Gis Élevage Demain, 118 p. https://www.gis-elevages-demain. org/Media/fichiers/Emplois-lies-a-1-elevagedans-le-grand-ouest.

Loyon L., Burton C.H., Misselbrook T., Webb J., Philippe F.X., Aguilar M., Doreau M. Hassouna M., Veldkamp T., Dourmad J.Y., Bonmati A., Grimm E., Sommer S.G., 2016. Best available technology for European livestock farms: Availability, effectiveness and uptake. J. Environ. Manage., 166, 1-11.

Mabon F., Raimbault T., Moreau P., Devienne S. Delaby L., Durand P., Ruiz L., Vertès F., 2009 Concilier efficacité technico-économique et environnementale des exploitations agricoles en zone vulnérable : apport du diagnostic agraire. Fourrages, 199, 373-388.

Menesguen A., 2003. Les « marées vertes » en Bretagne, la responsabilité du nitrate: Ifremer. http://archimer.ifremer.fr/doc/00000/143/.

Moreau P., Ruiz L., Mabon F., Raimbault T., Durand P., Delaby L., Devienne S., Vertès F., 2012. Reconciling technical, economic and environmental efficiency of farming systems in vulnerable areas. Agricult. Ecosys. Environ. $147,89-99$

Nutrinoe, 2015. La nutrition animale en Bretagne. http://www.nutrinoe.fr/.

Peyraud J.L., Cellier P., Aarts F., Béline F. Bockstaller C., Bourblanc M., Delaby L. Donnars C., Dourmad J.Y., Dupraz P., Durand P., Faverdin P., Fiorelli J.L., Gaigné C., Girard A., Guillaume, F., Kuikman, P., Langlais, A., Le Goffe P., Le Perchec S., Lescoat P., Morvan T., Nicourt C., Parnaudeau V., Rechauchère O. Rochette P., Vertes F., Veysset P., 2012. Les flux d'azote liés aux élevages : réduire les pertes, rétablir les équilibres. FRA : Inra - DEPE. http://prodinra.inra.fr/record/319012

Reise C., Musshoff O., Granoszewski K. Spiller, A., 2012. Which factors influence the expansion of bioenergy? An empirical study of the investment behaviours of German farmers. Ecol. Econ., 73, 133-141.
Roguet C., Gaigné C., Cariou S., Carlier M., Chatellier V., Chenut R., Daniel K., Perrot C., 2015. Spécialisation territoriale et concentration des productions animales européennes : état des lieux et facteurs explicatifs. INRA Prod. Anim. $28,5-22$.

Svoboda N., Taube F., Kluß C., Wienforth B., Kage H., Ohl S., Hartung E., Herrmann A., 2013. Crop production for biogas and water protection- trade-off? Agricult. Ecosys. Environ, 177, 36-47.

Thenail C., 2002. Relationships between farm characteristics and the variation of the density of hedgerows at the level of a micro-region of bocage landscape. Study case in Brittany, France. Agricult. Sys., 71, 207-230.

Thenail C., Joannon A., Capitaine M., Souchère V., Mignolet C., Shermann N., Pietro F., , Pons Y., Gaucherel C., Viaud V., Baudry J., 2009. The contribution of crop-rotation organization in farms to crop-mosaic patterning at local landscape scales. Agricult. Ecosys. Environ., 131, 207-219.

Thiering J., Bahrs E., 2011. Biogasproduktion in Deutschland - Sollte die energetische Nutzung von Wirtschaftsdünger explizit gefördert werden? Ger. J. Agr. Eco., 60, 259-275.

Weiland P., 2006. Biomass Digestion in Agriculture : A Successful Pathway for the Energy Production and Waste Treatment in Germany. Eng. Life Sci., 6, 302-309.

Weiland P., 2013. Production de biogaz par les exploitations agricoles en Allemagne. Sci. Eaux Territoires, 12, 14-23

Weiss F., Leip A., 2012. Greenhouse gas emissions from the EU livestock sector: a life cycle assessment carried out with the CAPRI model. Agricult., Ecosys. Environ., 149, 124-134.

Whiting A., Azapagic A., 2014. Life cycle environmental impacts of generating electricity and heat from biogas produced by anaerobic digestion. Energy, 70, 181-193.

Wilfart A., Espagnol S., Dauguet S., Tailleur A., Gac A., Garcia-Launay F., 2016. Ecoalim: a dataset of environmental impacts of feed ingredients used in french animal production. Plos One, 11 (12), e0167343. DOI : 10.1371/journal. pone. 0167343 .

zu Ermgassen E.K.H.J., Phalan B., Green R.E., Balmford A., 2016. Reducing the land use of EU pork production: where there's swill, there's a way. Food Policy, 58, 35-48. 


\title{
Résumé
}

Dans cet article, nous analysons trois territoires à haute densité animale, différentiés par le contexte de production (densité et diversité des élevages), la sensibilité des milieux et les choix stratégiques et technologiques mis en ouvre pour la gestion des effluents : $i$ ) la Bretagne, un territoire de polyculture-polyélevage avec une forte densité d'élevages et des zones particulièrement sensibles sur le plan environnemental, ii) l'Allemagne, avec la particularité d'un très fort développement de la méthanisation à des fins de production d'énergie renouvelable et iii) la Catalogne, un territoire marqué par un développement récent et très rapide de la production porcine dans un milieu où les surfaces agricoles disponibles pour l'épandage sont limitées. Ces systèmes se caractérisent par une production élevée par unité de surface et par unité de travail, à des coûts relativement bas, avec un recours important à des intrants, principalement pour l'alimentation des animaux. Malgré des progrès significatifs, les impacts environnementaux locaux, eutrophisation et acidification, constituent une limite importante dans ces systèmes. À l'inverse, les impacts globaux, comme l'émission de gaz à effet de serre sont faibles par unité de produit, compte tenu du type d'élevage et de l'intensité de production. Ils sont encore réduits avec la méthanisation. Ces systèmes font face à une remise en cause du modèle socio-économique dans lequel ils s'inscrivent avec des questions autour de la faible rémunération des éleveurs et du bien-être animal. Différents leviers d'action spécifiques à ces territoires à haute densité animale ont été identifiés pour mieux gérer les compromis entre les différents services.

\begin{abstract}
Diversity of service provided by territories with high density of animal production, three case studies

We analysed three case-study of European territories with highly dense animal production, and with different diversity of animal farming systems, sensitivity of their ecosystem, and strategies for manure management: i) Brittany, a mixed-farming territory with mixed-animal species, and highly sensitive ecosystems, ii) Germany a territory with intensive development of anaerobic digestion for the production of renewable energy and iii) Catalonia a territory with a recent and very fast development of pig production in a situation with limited agricultural land. These systems are characterized by high production levels per area and labor units, with an intensive use of external inputs, especially for animal feeding. In spite of significant progress, negative local environmental impacts, especially eutrophication and acidification, remains a critical point of these systems. Conversely, global impacts, such as the emission of greenhouse gases are low per production unit, because of the production type, mainly dairy and monogastric animals, and their high efficiency. They are even reduced for methane production. These systems are also facing socio-economic questioning in relation to highly fluctuating incomes of farmers, and animal welfare. Different levers for action specific of these high animal density territories are proposed to improve managing the tradeoffs between the different services they provide.
\end{abstract}

DOURMAD J.-Y., DELABY L., BOIXADERA J.-L., ORTIS C., MÉDA B., GAIGNÉ C., DUMONT B., 2017. Diversité des services rendus par les territoires à forte densité d'élevages, trois cas d'étude en Europe. In : Numéro spécial, L'élevage en Europe : une diversité de services et d'impacts. Dumont B. (Éd). INRA Prod. Anim., 30, 303-320. 
